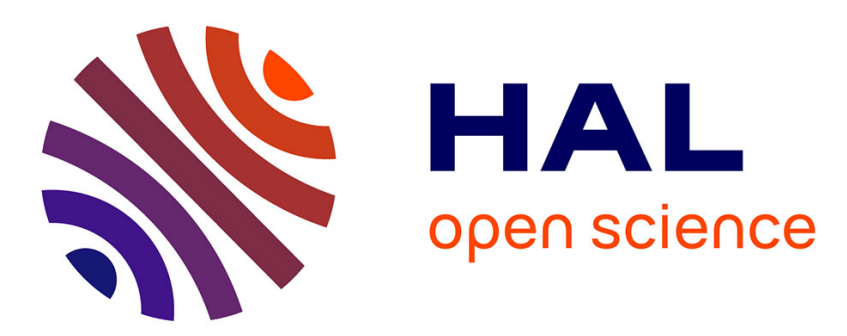

\title{
Experimental and numerical study of the plasma in coaxial capacitive coupled radio frequency discharge
}

Sara Alhomsi, Gérard Bauville, Stéphane Pasquiers, Tiberiu Minea

\section{To cite this version:}

Sara Alhomsi, Gérard Bauville, Stéphane Pasquiers, Tiberiu Minea. Experimental and numerical study of the plasma in coaxial capacitive coupled radio frequency discharge. Journal of Applied Physics, 2021, 130 (12), pp.123302. 10.1063/5.0060781 . hal-03364901

\section{HAL Id: hal-03364901 \\ https://hal.science/hal-03364901}

Submitted on 5 Oct 2021

HAL is a multi-disciplinary open access archive for the deposit and dissemination of scientific research documents, whether they are published or not. The documents may come from teaching and research institutions in France or abroad, or from public or private research centers.
L'archive ouverte pluridisciplinaire HAL, est destinée au dépôt et à la diffusion de documents scientifiques de niveau recherche, publiés ou non, émanant des établissements d'enseignement et de recherche français ou étrangers, des laboratoires publics ou privés. 


\section{AUTHOR QUERY FORM}

\begin{tabular}{|l|l|l|}
\hline & Journal: J. Appl. Phys. & $\begin{array}{l}\text { Please provide your responses and any corrections by } \\
\text { annotating this PDF and uploading it to AIP's eProof } \\
\text { website as detailed in the Welcome email. }\end{array}$ \\
\hline Publishing & Article Number: JAP21-AR-03314 & \\
\hline
\end{tabular}

Dear Author,

Below are the queries associated with your article; please answer all of these queries before sending the proof back to AIP.

Article checklist: In order to ensure greater accuracy, please check the following and make all necessary corrections before returning your proof.

1. Is the title of your article accurate and spelled correctly?

2. Please check affiliations including spelling, completeness, and correct linking to authors.

3. Did you remember to include acknowledgment of funding, if required, and is it accurate?

\begin{tabular}{|c|c|}
\hline $\begin{array}{l}\text { Location in } \\
\text { article }\end{array}$ & $\begin{array}{l}\text { Query / Remark: click on the } Q \text { link to navigate } \\
\text { to the appropriate spot in the proof. There, insert your comments as a PDF annotation. }\end{array}$ \\
\hline Q1 & $\begin{array}{l}\text { Please check that the author names are in the proper order and spelled correctly. Also, please ensure that each author's given } \\
\text { and surnames have been correctly identified (given names are highlighted in red and surnames appear in blue). }\end{array}$ \\
\hline Q2 & In the sentence beginning "However, the energetic ions...," please confirm that "the following sections" refers to Secs. III and IV. \\
\hline Q3 & $\begin{array}{l}\text { In the sentence beginning "For Subsections III A and III B...," please confirm that "the following sections" refers to } \\
\text { Subsections III A and III B. }\end{array}$ \\
\hline Q4 & Please reword the sentence beginning with "There is a huge interest" so that your meaning will be clear to the reader. \\
\hline Q5 & $\begin{array}{l}\text { We were unable to locate a digital object identifier (doi) for Refs. } 7,29 \text {, and } 33 \text {. Please verify and correct author names and } \\
\text { journal details (journal title, volume number, page number, and year) as needed and provide the doi. If a doi is not available, no } \\
\text { other information is needed from you. For additional information on doi's, please select this link: http//www.doi.org/. }\end{array}$ \\
\hline Q6 & Please provide publisher's name for Ref. 16 \\
\hline Q7 & $\begin{array}{l}\text { In Ref. } 32 \text {, please provide a brief description of the information available at the website. For example, "See XX for information } \\
\text { about XXX." }\end{array}$ \\
\hline Q8 & $\begin{array}{l}\text { In the text, variables are italic in some places, roman in others. Please check and change if you would like them to be set } \\
\text { consistently throughout your paper. }\end{array}$ \\
\hline
\end{tabular}


Please confirm ORCIDs are accurate. If you wish to add an ORCID for any author that does not have one, you may do so now.

For more information on ORCID, see https://orcid.org/.

Sara Alhomsi-0000-0002-1735-2235

Gérard Bauville

Stéphane Pasquiers

Tiberiu Minea-0000-0003-2886-3492

Please check and confirm the Funder(s) and Grant Reference Number(s) provided with your submission:

Please add any additional funding sources not stated above:

Thank you for your assistance. 


\title{
Experimental and numerical study of the plasma in coaxial capacitive coupled radio frequency discharge
}

\author{
Cite as: J. Appl. Phys. 130, 000000 (2021); doi: 10.1063/5.0060781 \\ Submitted: 22 June 2021 · Accepted: 23 August 2021 . \\ Published Online: $m-2021$
}

Sara Alhomsi, (iD Gérard Bauville, Stéphane Pasquiers, and Tiberiu Minea ${ }^{\text {a) }}$ (D)

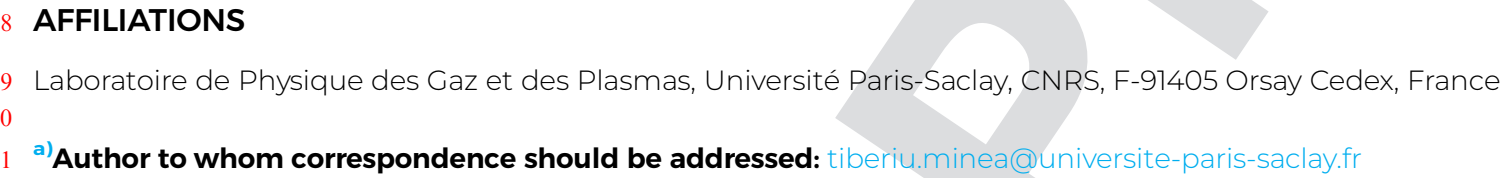

\begin{abstract}
A coaxial configuration of discharge is proposed for plasma surface treatment and possibly other applications. The reactor is based on a cylindrical structure, with the inner cylinder radio frequency powered (RF, $13.56 \mathrm{MHz})$ and the outer cylinder grounded, playing the role of a guard ring. The charged species can escape from the inner cavity through two longitudinal slits made in both cylinders, aligned to each other, and producing a linear slab of plasma. Hence, it is possible to project the plasma directly onto a surface placed under the slits, called external plate. The operation of this device is uniform and stable in argon for a large pressure range (0.8-50 mbar). Furthermore, simulations using the Plasimo ${ }^{\mathrm{TM}}$ software package were performed to evaluate the plasma parameters and to explain the experimental results. The ion flux on the surface exposed to this plasma increases when RF power increases, and the pressure or gap distance to the plate decreases. This cylindrical capacitive coupled plasma configuration can be very effective for surface treatment of different materials (conductors or insulators) on large area (when the plate or the system is moving perpendicular to the slits) due to energetic ions and active species released from the plasma.
\end{abstract}

Published under an exclusive license by AIP Publishing. https://doi.org/10.1063/5.0060781

\section{INTRODUCTION}

Radio frequency (RF) glow discharges have been intensively studied starting with the early 1960 s essentially driven by the microelectronics research. These plasmas involve a variety of physical and chemical processes, and they are particularly interesting for the large area applications. ${ }^{1}$ The biggest impact of RF is still in microelectronics where material processing is essential for very large-scale integrated (VLSI) circuits and high aspect ratio etching to make vias, but also it is extensively used for photovoltaic thin film deposition, for packaging treatment, and other plasma-based technologies. ${ }^{2}$

The capacitive coupled plasma (CCP) is the most popular RF discharge because the equipment is very simple, typically the one designed for direct current (DC) plasma but runs with radio frequency power.

Generally, the potential structure in the plasma tends to confine electrons and to expel positive ions through the oscillating sheaths, ${ }^{3}$ since at this frequency, the ions are sensitive only to the average potential due to their inertia.
The classic treatment of sheaths in RF discharges has been 44 well described and understood for more than 50 years. ${ }^{4}$ Koenig and 45 Maissel ${ }^{5}$ were the first to use the capacitive model of the sheaths to 46 explain the electrical behavior of the RF glow discharge systems for 47 RF sputtering. Briefly, the magnitude of the sheath capacitances at 48 the powered electrode $\left(C_{p}\right)$ and the (grounded) wall $\left(C_{g}\right)$ is deter- 49 mined primarily by the relative area of the excitation electrode $\left(A_{p}\right) \quad 50$ and grounded surfaces $\left(A_{g}\right)$ in contact with the glow discharge. ${ }^{7} \quad 51$ Depending on the operation pressure, several regimes can be found 52 for the transport of charged species through the sheath. Hence, it 53 was established that the ratio of the DC voltage dropping on each one of the sheaths (facing the two electrodes) is inversely proportional to the electrode area ratio to the power $n$, where $n$ lies between 1.2 and $4{ }^{6}$

Laboratory and industrial plasma sources are often geometrically asymmetric, i.e., the surface areas of the powered and grounded electrodes $\left(A_{p}\right.$ and $\left.A_{g}\right)$ are not equal, leading to the generation of a DC self-bias voltage to ensure equal fluxes of positive and negative charges in average, over the RF period. For capacitive 
63 coupling, there is no net conduction current flowing through the 64 circuit, neither on the excitation electrode circuit nor on the 65 grounded one. Therefore, the plasma potential must approach the 66 potential of the excitation electrode for a brief period during each $67 \mathrm{RF}$ cycle to allow electrons reaching the excitation electrode. 68 Similarly, the plasma potential must also approach ground poten69 tial for a brief period during each cycle. Therefore, the expression 70 of the self-bias $\left(V_{\text {bias }}\right)$ for capacitively coupled systems ${ }^{7}$ is given by 71 the following equation:

$$
V_{\text {bias }}=V_{R F}\left(\frac{C_{p}-C_{g}}{C_{p}+C_{g}}\right)
$$

72 where $V_{R F}$ is the amplitude of applied RF voltage.

Due to extensive use in the semiconductor industry, planar asymmetric plasma reactors are relatively well understood. Early works on the geometrically asymmetric RF capacitive discharges were focused on planar excited electrode and a large area grounded (often the discharge chamber itself), which generally had a cylindrical shape. ${ }^{7-9,12-16}$

On the contrary, linear RF plasma for surface treatment is much rarer. It has been recently proposed, for instance, by Baránková et al. ${ }^{17}$ This configuration was successfully exploited for plasma enhanced chemical vapor deposition (PECVD) combined with physical vapor deposition (PVD) and efficiently operated with a hallow powered electrode (target) formed of two parallel (graphite) plates. However, they did not follow or reported on the self-bias voltage on the RF electrode and the role of ions in this type of device. They choose to use a second RF power supply to bias the dielectric (glass) substrate in order to control the ion flux.

89 electrodes operated with a single RF power supply. Let us note that only a few experiments and models do exist for this case. ${ }^{18,19}$ 2 Recently, Upadhyay et al. ${ }^{19}$ used an asymmetric non-planar geometry 3 to process the inner walls of superconductive radio frequency (SRF) 4 cavities. They powered the inner cylinder of the cylindrical capacitive 5 coupled discharge (CCCD), while the outer cylinder was grounded, 6 which was, in their case, the cavity wall to be etched. The plasma, in 97 their case, develops between the two cylinders. The surface area asym8 metry between inner and outer electrodes (cylinders) creates a nega9 tive self-bias potential on the inner electrode, obviously smaller.

In the present work, the novel design of CCCD is proposed, and it succeeded to produce a linear plasma that survives inside the 02 inner (powered) electrode but can also escape through a slit to 103 treat, for instance, an external plate placed under the reactor 104 (facing the slit). In this work, the gas used is argon. The plasma 105 formed in the cylindrical coaxial cavity is stable over a large range 106 of pressures $(0.8-50 \mathrm{mbar})$ and RF powers $(20-1200 \mathrm{~W})$. We tested 107 the operation of the initial configuration, called "reactor A," and 108 aiming its scaling-up, a second configuration has been tested after109 ward with longer cylinders, called "reactor B."

In this paper, we present the proposed configuration of $\mathrm{CCCD}$, powered by a radio frequency power supply operating at $13.56 \mathrm{MHz}$, and the optimization of the system in terms of the production of charged particles close to the plate. We report the main plasma parameters obtained by combining the experimental and 114 numerical approaches.

Section II describes the experimental setup and the numerical 116 approach. The experimental results for both reactors A and B are 117 presented in Sec. III. In Sec. IV, the numerical findings are pre- 118 sented, and the experimental results are discussed in light of these 119 simulations. Finally, Sec. V summarizes the main achievements and 120 the conditions for optimizing the operation of the proposed CCCD 121 configuration.

\section{EXPERIMENTAL SETUP AND NUMERICAL APPROACH}

\section{A. Experimental setup and diagnostics}

A new reactor has recently been developed to generate a linear 126 slab of plasma with the control of the ion species (energy and 127 density) using a single RF power supply (see Figs. 1 and 2). The 128 CCCD reactor is formed by two coaxial tubes of different radii, 129 each one equipped with a longitudinal slit. The edges were closed 130 by two stainless-steel supports. They ensure perfect parallelism 131 between the two cylinders; the spacer they contain is made of 132 dielectric material (alumina) and the RF is fed by one side. Only 133 the inner cylinder is powered, the outer being grounded. 134

The first characterization was performed with an initial config- 135 uration, the so-called reactor "A." This configuration has the RF elec- 136 trode made of stainless-steel $\left(90 \mathrm{~mm}\right.$ long, $r_{\text {in }}=5 \mathrm{~mm}$ inner radius 137 and $r_{\text {out }}=6.4 \mathrm{~mm}$ outer radius) with the slit area of $50 \times 2 \mathrm{~mm}^{2} 138$ (length $\times$ width $=L_{\text {in }} \times w_{\text {in }}$ ). The outer cylinder was made of copper 139 ( $120 \mathrm{~mm}$ long, $R_{\text {in }}=8 \mathrm{~mm}$ inner radius and $R_{\text {out }}=10 \mathrm{~mm}$ outer 140 radius), with a slit area of $60 \times 4 \mathrm{~mm}^{2}$ (length $\times$ width $\left.=L_{\text {out }} \times w_{\text {out }}\right) . \quad 141$

The essential choice for our work was the geometry of metal 142 electrodes, but the materials itself does not have a significant effect 143 on the various experimental studies carried out.

The distance between these tubes is $\delta=1.6 \mathrm{~mm}$, and the two 145 slits are aligned facing each other (see Fig. 2). 146

The role of the external cylinder is to avoid the plasma forma- 147 tion on the outer surface of the powered electrode, forcing the 148 operation of the system in a hollow electrode mode. The distance $\delta 149$ between the two cylinders is one of the critical parameters and 150 must be made as small as possible. Hence, the outer cylinder oper- 151 ates as a guard ring, and no plasma (or a very weak one) develops 152 between the two cylinders.

When RF power is applied, the plasma glows inside the inner 154 tube cavity, but it leaks out through the slits and spreads onto the 155 surface of the external plate, here copper, placed just under the 156 slits. The gap separating the outer cylinder and the plate is denoted 157 as $g$, with $g=8 \mathrm{~mm}$ (see Figs. 1 and 2). This plate was also 158 grounded, but it is possible to bias it as desired, positively or nega- 159 tively, depending on the followed purpose.

This system is very compact, and the plasma stays confined in 161 a limited volume between the CCCD reactor and the plate. The 162 system was placed in a vacuum chamber of $45 \mathrm{~cm}$ height and $35 \mathrm{~cm} 163$ diameter, made of stainless-steel. Prior to each experiment, the 164 primary vacuum was obtained via a Pfeiffer duo 004 (pumping 165 speed $4 \mathrm{~m}^{3} / \mathrm{h}$ ) pump, reaching the base limit pressure of $10^{-2}$ mbar. 166 Then, the argon gas was injected, over a pressure range lying 167 between 0.8 and 50 mbar. The pressure was given by an absolute 168 


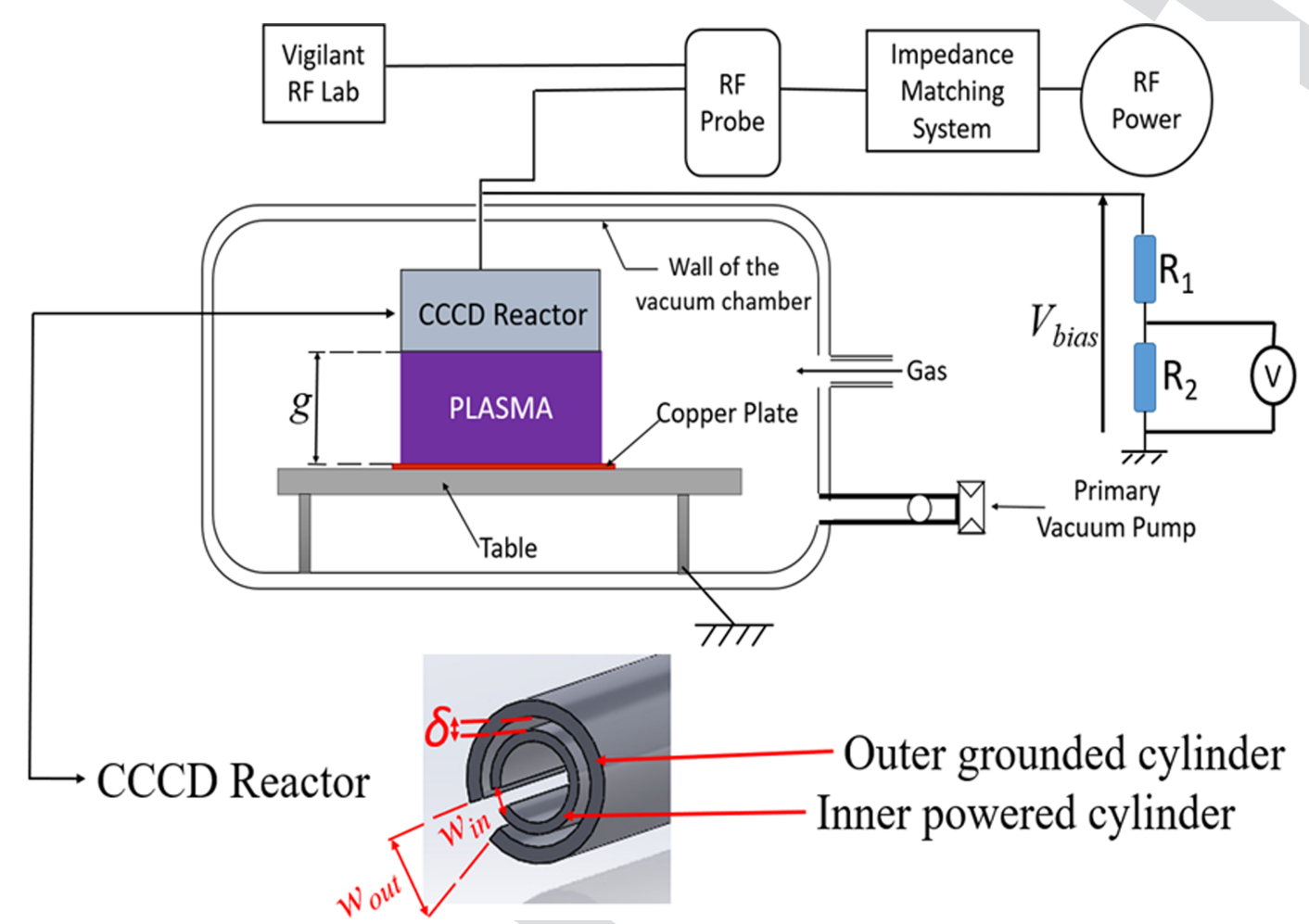

FIG. 1. Sketch of the experimental setup. $p=1.6 \mathrm{~mm}$ is the distance between the cylinders, $w_{\text {in }}=2 \mathrm{~mm}$ and $w_{\text {out }}=4 \mathrm{~mm}$ are the widths of the slits for inner and outer cylinder, respectively.

169 capacitive gauge (Pfeiffer CMR 361). The glow discharge was estab-

170 lished by applying the RF delivered by the power supply (COMET,

$17113.56 \mathrm{MHz}, 3 \mathrm{~kW})$ to the inner electrode. It was connected to an

172 automatic impedance matching system to ensure the impedance

173 tuning.

174 So, the potential (boundary conditions) on each electrode is as

175 follows.
On the inner electrode:

$$
V_{R F}(t)=V_{\text {bias }}+V_{M A X} \sin (\omega t) .
$$

On the outer electrode and the plate:

$$
V_{g}=0 .
$$

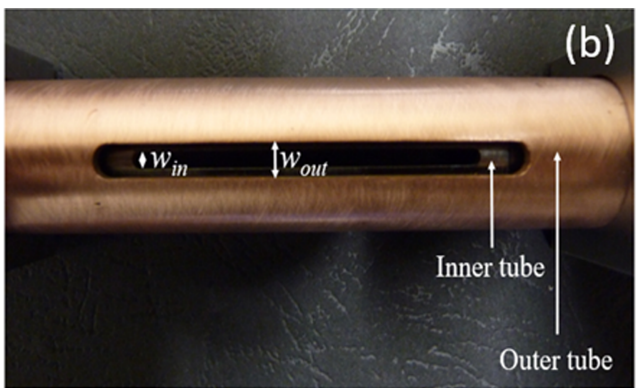

FIG. 2. Illustration of (a) the experimental arrangement of $\operatorname{CCCD}$ and (b) the view showing the slits of the tubes; $\mathrm{w}_{\text {in }}=2 \mathrm{~mm}$ and $\mathrm{w}_{\text {out }}=4 \mathrm{~mm}$ are the widths of the slits for inner and outer cylinder, respectively. 
The circuit includes an RF probe (Vigilant Power Monitor181 SOLAYL), which measures the electrical parameters (average 182 power, voltage amplitude, maximum current, phase shift, imped183 ance, harmonics, etc.). It is driven by a software (Vigilant RF Lab) 184 that also allows us to display the measured data. However, this 185 probe is unable to measure the DC voltage (self-bias) developed on 186 the powered electrode because the voltage sensor is "AC coupled" 187 to the RF electrode. For this reason, we measured independently 188 the self-bias voltage by a home-made system described below.

Note that the absolute values of self-bias are displayed on the power supply but not its sign. The measurements were performed using a blocking capacitor, which excludes the effective DC ion current in the circuit. In fact, this capacitor is one of the capacitances of the impedance matching system. To precisely know the sign of the DC self-bias, we also measured this voltage using a voltmeter. The voltage drop onto one of the resistances of a voltage divider circuit is measured placing the divider between the RF fed line and the ground, as close as possible of the powered electrode. Notice that the electrical connection was still out of the vacuum chamber, at atmospheric pressure (see Fig. 1). The divider consists of two huge resistors $\left(R_{1}\right.$ and $\left.R_{2}\right)$ in series with $R_{1}=R_{2}=10 \mathrm{M} \Omega$, such as the current leakage through this branch of the circuit could be neglected with respect to the discharge one. The voltage measurement is performed over the second resistor $\left(\mathrm{R}_{2}\right)$, and the selfbias voltage is obtained, obviously, multiplying this voltage by 2 (Fig. 1). It should also be noted that the difference between the voltage returned by the generator and the absolute value of the voltage measured by the voltmeter (multiplied by two) is about $14 \%$, this difference being due to the position on the RF circuit between the two measurement points.

A similar configuration was tested but with longer cylinders/ slits, reactor B: inner cylinder was made also of stainless-steel $180 \mathrm{~mm}$ long, with a slit area of $145 \times 2 \mathrm{~mm}^{2}$. The corresponding outer cylinder was made of cooper, $210 \mathrm{~mm}$ long, and with a slit area of $150 \times 4 \mathrm{~mm}^{2}$. The plasma develops as expected in a similar way, linearly, all along the slits leading to a plasma slab outside, toward an external plate facing the slits. Beyond the influence of the RF power or the gas pressure on the CCCD, it is very interesting to study the effect of the gap distance $(g)$ to the plate. Hence, the gap for configuration $\mathbf{B}$ of this reactor was varied between 1 and $10 \mathrm{~mm}(g=1,6$, and $10 \mathrm{~mm})$.

\section{B. Numerical approach}

For the numerical study of the geometrically asymmetric discharge system, the MicroDischarge model of Plasimo package software has been used. ${ }^{32}$ It is based on the fluid approach to model the plasma. A detailed description of the model can be found in Ref. 20 and references therein. Hereafter, only the basic features of this model, in particular, the species and reactions, are presented as well as the numerical approximation for this specific discharge geometry.

\section{Model equations}

Following the conventional fluid approach, the MicroDischarge model of Plasimo package solves the conservation equations system, derived from the moments of the Boltzmann transport equation, and coupled with the Poisson equation for the 234 charged species. These balance equations were solved for a number 235 of species using the drift-diffusion approximation. The species 236 included in the model, such as electrons, ions, and excited atoms, 237 are described in more detail in Sec. II B. The conservation equa- 238 tions for the different species are

$$
\frac{\partial n_{p}}{\partial \mathrm{t}}+\nabla \cdot \Gamma_{p}=S_{p},
$$

with $\Gamma_{p}$ being the drift-diffusion flux,

$$
\Gamma_{p}= \pm \mu_{p} E n_{p}-D_{p} \nabla n_{p},
$$

where $n_{p}$ is the number volume density of species $p, S_{p}$ is the net 241 source term of species $p$ due to reactions induced by the plasma 242 kinetics, $\mu_{p}$ and $D_{p}$ are the mobility and the diffusion coefficient of 243 species $p$, respectively, and $E$ is the local electric field.

In order to specify the local variation of the reaction rate coef- 245 ficients and electron transport coefficients as a function of the 246 mean electron energy, an additional balance equation for electron 247 energy was included:

$$
\frac{\partial\left(n_{\mathrm{e}} \varepsilon_{\mathrm{e}}\right)}{\partial t}+\nabla \cdot \Gamma_{\varepsilon_{\mathrm{e}}}=S_{\varepsilon_{\mathrm{e}}}
$$

in which $\varepsilon_{\mathrm{e}}$ is the average electron energy and $n_{e}$ is the electron 249 number volume density. The source term of this equation, $S_{\varepsilon_{\mathrm{e}}}$, rep- 250 resents the net source term of energy for the plasma electrons, i.e., 251 the energy gained by the electric field and lost by the collisions. 252 The electron energy flux $\Gamma_{\varepsilon_{\mathrm{e}}}$ is described in Ref. 20,

$$
\Gamma_{\varepsilon_{\mathrm{e}}}=-\frac{5}{3} \mu_{e} E n_{e} \varepsilon_{\mathrm{e}}-\frac{5}{3} n_{e} D_{e} \nabla \varepsilon_{\mathrm{e}} .
$$

The electron transport and reaction rate coefficients were pre- 254 calculated by a Boltzmann solver-Bolsig+, ${ }^{21}$ for an equilibrium 256 electron energy distribution function at different reduced electric 257 fields. The resulting data set was used to create a lookup table to be 258 used as input for the Plasimo model. The transport coefficients for 259 ions were extracted from the LXCat Database ${ }^{22}$ and were used as 260 input for the model as a function of the reduced electric field. For 261 the charged particles, the diffusion coefficients were calculated 262 from the mobilities using Einstein' relation. ${ }^{31} 263$

The constant diffusion coefficient for the excited species was 264 taken from Ref. 23.

Finally, in addition to the balance equations discussed above, 266 the Poisson equation was solved,

$$
\nabla \cdot\left(\epsilon_{p l} \nabla \varphi\right)=-\nabla \cdot\left(\epsilon_{p l} E\right)=-\sum_{p} q_{p} n_{p}
$$

in which $\varphi$ is the electric potential as a function of position and 268 time, $\epsilon_{p l}$ is the permittivity of the medium, and $q_{p}$ is the charge of 269 the species $p$. From this, the electric field and potential distribution 270 in the discharge were determined in a cross section (2D) perpen- 271 dicular to the cylinder's axis. All equations of the model are solved 272 
TABLE I. Species and reactions included in the model, with references to the original literature from which the transport coefficients for particles or rate coefficients were considered.

\begin{tabular}{lccc}
\hline \hline Species & Transport coefficient (Ref.) & Reactions & Rate coefficient (Ref.) \\
\hline $\mathrm{E}$ & Bolsig+ (Ref. 21) & $\mathrm{Ar}+\mathrm{e} \Leftrightarrow \mathrm{Ar}^{+}+$2e & Bolsig+ (Ref. 21) \\
$\mathrm{Ar}+$ & LXCat (Ref. 22) & $\mathrm{Ar}+\mathrm{e} \Leftrightarrow \mathrm{Ar}+\mathrm{e}$ & Bolsig+ (Ref. 21) \\
$\mathrm{Ar}^{*}$ & (Ref. 23) & Elastic energy loss & Bolsig+ (Ref. 21) \\
\hline \hline
\end{tabular}

273 by the finite difference method, where all quantities are represented 274 by their values at a discrete set of points in time and space. ${ }^{20}$

\section{Species and reactions}

In this work, we run the code for argon gas, by using the corresponding reaction rates and transport coefficients of particles, as described in Subsection II C. Three species have been considered in the model: electrons (e), single charged positive ions $\left(\mathrm{Ar}^{+}\right)$, and one effective excited state $\left(\mathrm{Ar}^{\star}\right)$.

At the walls, the incoming heavy particles were either neutralized if they are charged or de-excited if they are excited (i.e., the wall is fully absorber in terms of charge or energy). The resulting ground state atoms are assumed to feed the background gas, which is thus constant (unaffected by the plasma species). However, the energetic ions impinging the surfaces can yield to secondary electron emission at the wall (especially RF powered electrode, as detailed in Secs. III and IV). The secondary emission coefficients for $\mathrm{Ar}^{+}$depend strongly on the properties of the surface and are not exactly known. We used the value of 0.04 for $\mathrm{Ar}^{+}$induced secondary electron emission. More details on the boundary conditions of the model can be found in Ref. 20.

The transport coefficients for particles and the set of reactions between the species that were included in the model are summarized in Table I.

Note that we focused here on the spatial distribution of the density of charged particles, the electric field and potential, but not particularly on the detailed description of the excited states and gaseous kinetics. The latter are interesting particularly in the case of PECVD use of the CCCD, which is out of the scope of the present work. Hence, for the sake of simplicity, the excited levels are all reduced to one effective state to account on the energy loss of electrons in their inelastic path, considering the sum of the cross sections of all excited states.

A second approximation used in these calculations is to neglect the ionization from excited state. After several test simulations, it comes out that the average electrons energy is always greater than $4 \mathrm{eV}$, which confirms our second approximation.

\section{E. Discharge geometry}

The discharge structure is assumed to be infinite long, such as it could be reduced to $2 \mathrm{D}$ simulation geometry, with the cross section corresponding to the plane perpendicular to the cylinder's axis. The geometry was kept as close as possible to the real discharge system, with inner tube 5 and $6.4 \mathrm{~mm}$ inner and outer radii, respectively, with $2 \mathrm{~mm}$ width slit $\left(w_{i n}\right)$ (blue tube in Fig. 3) powered by the RF signal (10 MHz in simulation). The outer tube has $8 \mathrm{~mm}$ inner radius and $4 \mathrm{~mm}$ width slit $\left(w_{\text {out }}\right)$ (cyan in Fig. 3 ), 317 and it is connected to the ground together with the plate under the 318 slits. With these dimensions, the space between the two cylinders 319 $\delta=1.6 \mathrm{~mm}$, like in the experiment.

The axes $y$ and $z$ are defined as follows (see Fig. 3). The origin 321 $(0,0)$ is the point on the plate crossed by the normal to it passing 322 by the center of the slits (and the axis of the two cylinders). $y$ axis 323 is parallel to the plate surface and perpendicular to the cylinder's 324 axis. Positive and negative values describe the position on each side 325 of the slit (center). $z$ axis represents the vertical axis from the 326 center $(0,0)$ toward the coaxial electrodes structure, on which the 327 distance between the plate and the outer tube represents the gap $g$. $\quad 328$

The sketch of the simulation geometry is shown in Fig. 3. The 329 initial condition for the seed electrons and ions considers a homo- 330 geneous density of $10^{15} \mathrm{~m}^{-3}$. The metastable state (effective excited 331 state) density has been set to an initial density of $10^{7} \mathrm{~m}^{-3}$. These 332 input values have been changed several times to check their impact 333 on the final solution, and they were the highest without any impact 334 on the final result. So, they have been further systematically used 335 because the highest the input the faster the convergence of the 336 code. The numerical convergence had been considered achieved 337 after more than $60 \mu \mathrm{s}$ of real time simulation (600 RF periods), and 338 the average plasma parameters are certainly not evolving (moment 339

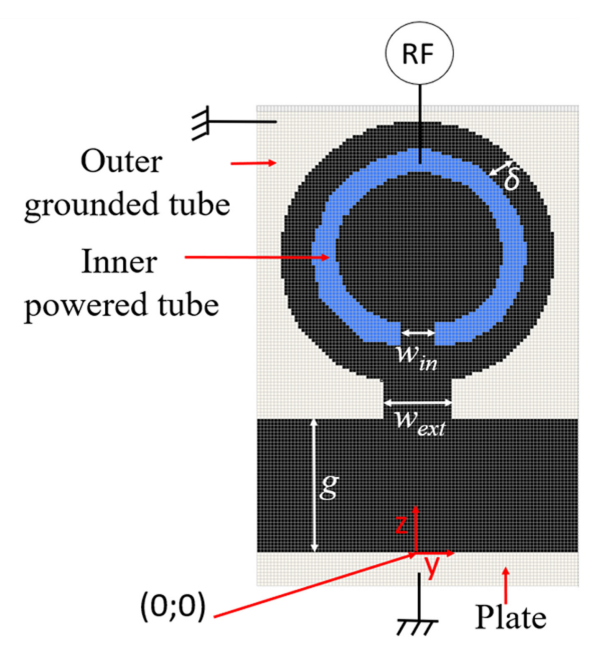

FIG. 3. Sketch of simulation geometry. Plasma volume is the black region. Inner electrode is the blue region and outer the cyan region (only the inner radius of the outer electrode is important). Cell size (in red) was $0.2 \times 0.2 \mathrm{~mm}^{2}$, $\delta=1.6 \mathrm{~mm}, \mathrm{w}_{\text {in }}=2 \mathrm{~mm}, \mathrm{w}_{\text {out }}=4 \mathrm{~mm}$, and $\mathrm{g}=8 \mathrm{~mm}$. 

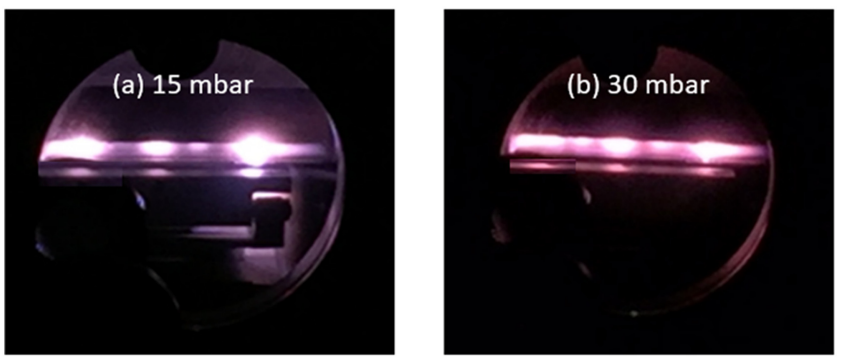

FIG. 4. Ar plasma images taken with the reactor $\mathbf{A}$ in operation for (a) 15 and (b) 30 mbar. Plasma power was kept constant at $200 \mathrm{~W}(\mathrm{~g}=8 \mathrm{~mm})$.

340 to moment in two consecutive RF periods) since, at least, $20 \mathrm{RF}$ 341 periods.

\section{EXPERIMENTAL RESULTS AND DISCUSSION}

For our CCCD configuration, $A_{p}$ represents the area of the 344 inner powered cylinder. The effective grounded electrode is a 345 certain area of the planar surface of the external plate together with 346 the inner area of the outer electrode if a (weak density) plasma 347 forms in the space between the two cylinders. If the plasma stands 348 out of the space between the cylinders, the grounded area that 349 accounts for the discharge $\left(A_{g}\right)$ is not given, obviously, by the whole 350 size of the plate, but only by the region covered by the plasma, 351 under and close to the slits. This area is defined by the surface 352 "seen" by the plasma slab escaping from the slit on the plate, i.e., 353 receiving charged species to close the RF electric circuit. It is not 354 easy to estimate it before running the experiments or numerical 355 simulations.

356 Hereafter, we explore the operation range of the discharge, the 357 stability of the plasma for different gas pressures, and RF powers.

358 For each case, the electrical characteristics of the discharge and the

Q3 359 self-bias voltage are reported. For Subsections III A and III B, we 360 have selected the main experimental results for the cases that have 361 been simulated in Sec. IV.

\section{A. First characterization (reactor A)}

This section presents the first experimental investigations of 363 the novel configuration. The typical operation has been found for a 364 pressure range between 15 and 50 mbar. For this set of results 365 reported here, the gap between the reactor and the plate was fixed 366 $(g=8 \mathrm{~mm})$. Systematic measurements have been performed varying 367 the RF power $\left(P_{R F}\right)$ and the gas pressure $(p)$ for argon plasma. 368 A linear plasma was observed all along the slit.

As said, when applying the RF power to the system, a dis- 370 charge glows and escapes out of the slits. Striations have been 371 observed along the slit. They are mobile in both directions between 372 the tube edges. The velocity and the number of striations strongly 373 depend on gas pressure (see Fig. 4) and RF power. Further studies 374 of these structures are very desirable, as recently reported by Bera 375 et $a l^{42}$ The striation discharge pattern is characterized by several 376 plasma "bubbles," alternating bright and dark regions, formed 377 outside the slit, in the gap between the electrodes and the plate. 378 The formation of such striations was already reported in direct 379 current (DC) glow discharges. ${ }^{24,26-28}$ For RF capacitive coupled 380 discharges, striations have also been also observed, in electronega- 381 tive gases ${ }^{25}$ and in argon, when plasma is created between two 382 wires wrapped around a long dielectric tube, over a certain range of 383 frequencies and gas pressures. ${ }^{29,30}$ The non-linear dependence of 384 the ionization rate on the electron density is shown to be the main 385 underlying mechanism of the striation phenomena. ${ }^{34} 386$

\section{Effect of gas pressure and RF power}

387

In this section, the effect of the gas pressure and RF power on 388 discharge parameters are presented, such as the voltage and current 389 amplitude $\left(V_{M A X}, I_{M A X}\right)$, the phase between voltage and current 390 $(\phi)$, the discharge impedance $(Z)$, and the self-bias voltage $\left(V_{\text {bias }}\right) .391$ They were measured for three pressures: 15, 30, and 50 mbar. As 392 expected, the gas pressure influences the electrical characteristics of 393 the RF discharge, as found before. ${ }^{35-38}$

Figure 5(a) shows the temporal evolution of $V_{\text {MAX }}$ and $I_{\text {MAX }} 395$ for each operation pressure. The discharge is stable for different 396 pressures and one can see that $V_{M A X}$ and $I_{M A X}$ are the highest for 397 the lowest pressure, here 15 mbar.

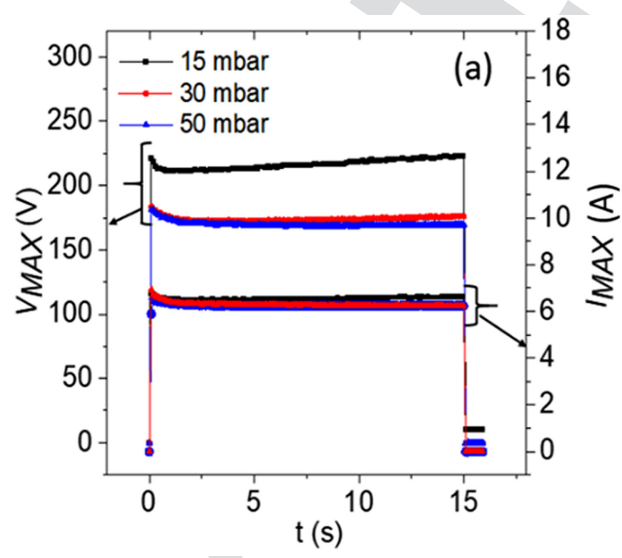

FIG. 5. (a) Temporal evolution of the voltage and current amplitude $\left(\mathrm{V}_{\text {MAX, }}\right.$ $I_{\text {MAX) for }} 15,30$, and 50 mbar. (b) Variation of the phase $(\phi)$ and the impedance (Z) with gas pressure. Plasma power was kept constant at $200 \mathrm{~W}$. 


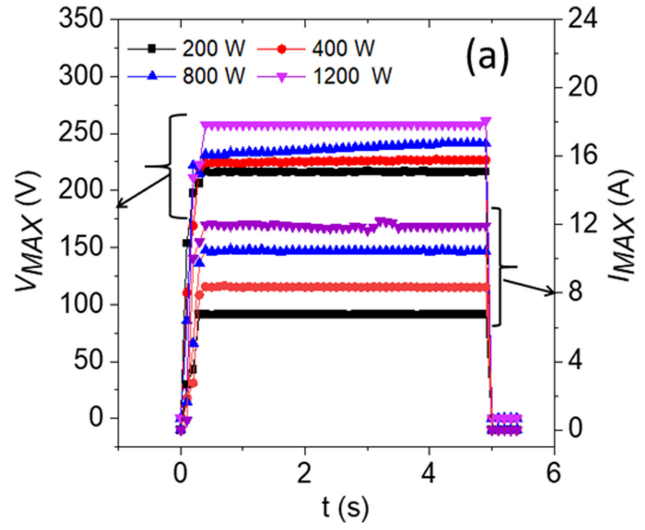

For 15 mbar, the slow drift of $V_{M A X}$ and $I_{M A X}$ from 0 to $15 \mathrm{~s}$ 400 is related to the heating of the electrodes, which is less important at 401 higher pressure, 30 and 50 mbar.

Figure 5(b) shows the variation of the phase and the impedance measured with the gas pressure. Obviously, the impedance and the phase (in absolute value) decrease with the gas pressure. ${ }^{35,38}$ The same effect of gas pressure on electrical characteristics is widely found for RF discharges. The discharge current decreases with increasing of pressure due to the increasing of electron-neutral collision frequency. The discharge voltage varies almost proportionally to the discharge current, and, therefore, the impedance remains in the narrow range contained in the interval (27.5-33 $\Omega$ ). The higher collision rates for higher pressures cause the increase of the ohmic (or resistive) component of the discharge, which yields to the decrease of the phase. ${ }^{35,38}$

The plasma impedance is the complex sum of resistive $(R$, real) and capacitive reactive ( $X$, imaginary) parts. Figure 6 shows the time evolution of different electrical characteristics $\left(V_{M A X}\right.$, $I_{M A X}, \phi, Z, X$, and $R$ ) but this time for several RF powers for a fixed operation pressure ( 15 mbar). It should be noted that $X$ is negative, but the absolute values are presented.

Figure 6(a) shows the temporal evolution of $V_{M A X}$ and $I_{M A X}$ for different RF powers $200,400,800$, and $1200 \mathrm{~W}$. The discharge is stable for different powers and one can see that $V_{M A X}$ and $I_{M A X}$ increase with RF power, as expected.

But, as shown in Fig. 6(b), the increase of the power is not only a result of the increasing voltages and currents but also due to the decreasing phase angles $(\phi)$, which means that the discharge becomes more resistive, and more power is dissipated by ohmic heating. This increase of the real part of the impedance turns the phase angle to smaller values. The physics of the increasing resistivity can be understood considering two different power dissipation channels in the discharge, following the model presented in Refs. 39 and 40: plasma resistivity is the sum of the ohmic resistance in the bulk (electron-neutral collisions) and of the resistance that models the power dissipation in the sheath (by acceleration of ions by the electric field in sheaths). The first decreases but the second increases more quickly with the RF power.

Obviously, the impedance decreases as the power increases [Fig. 6(b)]. This decrease is due essentially to that of the absolute value of the capacitive reactance $X$, i.e., a compression of the 439 sheaths thickness.

On the other hand, Fig. 7 shows the variation of the self-bias 441 (in absolute value) with the pressure at 100 and $200 \mathrm{~W}$. For all pres- 442 sures, the measured self-bias was negative. One can see that for 443 both RF powers, the self-bias is the highest for the lowest pressure, 444 here 15 mbar, in line with the finding for $V_{\text {MAX }}$ [Fig. 5(a)]. As 445 expected, its value increases with the power, $V_{\text {bias }}$ being higher at 446 200 than at $100 \mathrm{~W}$. Similar variation trends of the absolute value of 447 $V_{\text {bias }}$ with RF power and gas pressure were reported in many refer- 448 ences, see for instance Refs. 10, 11, and 19.

As already known, the increase of self-bias with RF power is 450 essentially due to the increase of the RF voltage [see Eq. (1)], at 451 constant power.

Hytry and Boutard-Gabillet ${ }^{10}$ and Upadhyay et al. ${ }^{19}$ have 453 explained the increase of the negative self-bias when decreasing the 454 pressure by the expansion of the plasma volume toward the 455

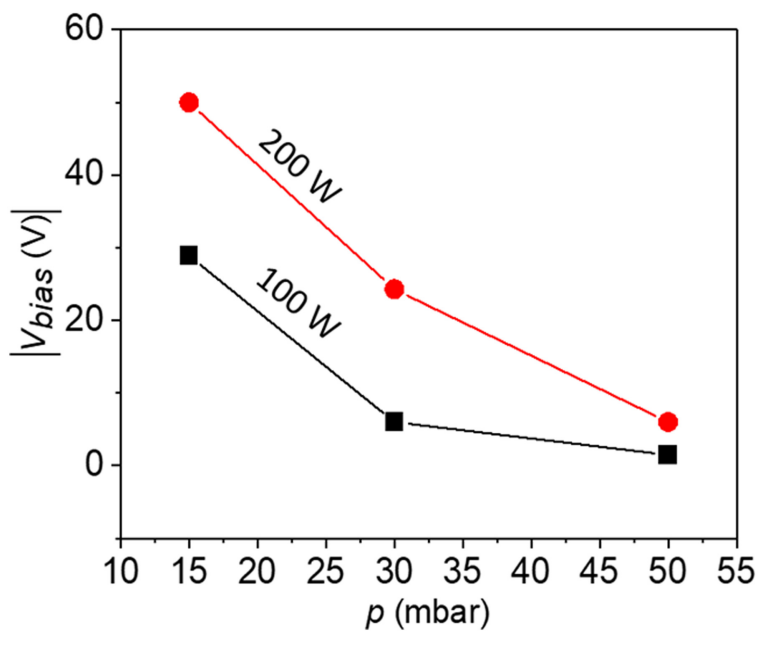

FIG. 7. Self-bias voltage (absolute value) variation with pressure, at 100 and $200 \mathrm{~W}$ RF power $(\mathrm{g}=8 \mathrm{~mm})$. 
456 grounded area. They verified experimentally that the sheath thick457 ness increases also with the pressure decrease. ${ }^{10}$ For our design of 458 CCCP, this plasma feature is discussed in detail in Sec. IV A.

\section{B. Operation with long cylinders (reactor B)}

According to the results obtained with the initial configuration of the reactor (Subsection III A-reactor A), a similar one has been built and tested, but with about twice longer slit (reactor B, Fig. 8). Hence, it is expected to enlarge the active region of the plasma. This allows the treatment of larger surfaces, keeping in view its possible application.

However, as the RF power is injected at one end of the inner cylinder, it is necessary to first check if the discharge glows all along the slit and not only close to the feeding side. As expected, the plasma glows in a similar way as for the shorter electrodes system (reactor A). Therefore, the scaling-up of the system seems well possible, and larger systems can be imagined, depending on the requirements.

Let us mention that the striation phenomenon was found again. However, one can easily remark the almost homogeneous light emission all along the slit at the highest pressure tested here, 9 mbar, especially in front of the plate where no variation of the emission light intensity could be recorded.

Concerning the parametric study, the same trends have been observed, as for the configuration A, for pressures between 15 and
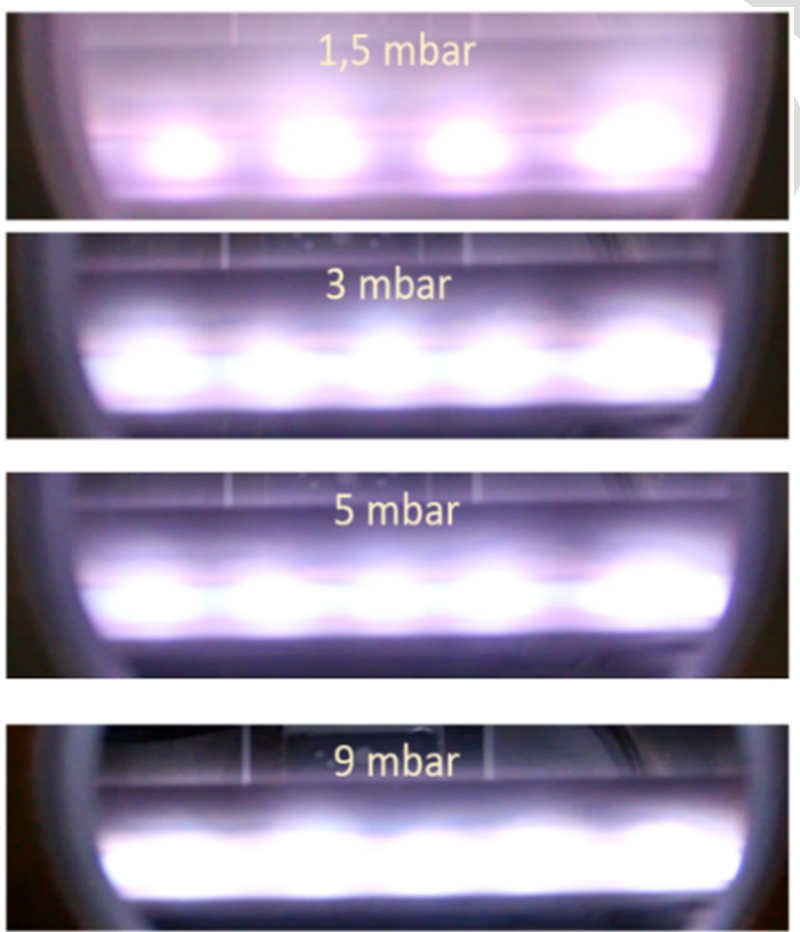

FIG. 8. Ar plasma images taken with the reactor $\mathbf{B}$ in operation for different pressures $\left(P_{R F}=20 \mathrm{~W}, \mathrm{~g}=10 \mathrm{~mm}\right)$.
50 mbar. However, this range has been extended toward the lower 480 pressures with the reactor $\mathrm{B}$, and a second study was carried out 481 for $p<15$ mbar.

Another interesting parameter varied in this section was the 483 reactor/plate gap distance $(g=1,6$, and $10 \mathrm{~mm})$.

\section{Effect of the reactor/plate gap}

The self-bias is plotted for different values of $g$ for Ar plasma 486 $(g=1,6$, and $10 \mathrm{~mm}$, see Fig. 9) vs the operation pressure. The 487 remarkable difference induced by the gap on the self-bias voltage 488 starts for pressures higher than 3 mbar. The self-bias voltage 489 increases, in absolute value, with the reduction of the gap, being 490 the highest for $g=1 \mathrm{~mm}$. For lower pressures ( $<3 \mathrm{mbar}$ ), the self- 491 bias voltage seems to be independent of the gap separating the 492 CCCD from the plate, which is interesting, again, for applications. 493

One expects that getting closer the plate to the slits of tubes, 494 the plasma density increases bringing more energetic particles 495 onto the plate. However, the recorded results seem to be consistent 496 in light of numerical simulations, presented and discussed in 497 Sec. IV B.

Figure 10 shows the maxima of RF voltage, RF current, and 499 self-bias voltage measured for 0.8 and $5 \mathrm{mbar}(g=6 \mathrm{~mm})$ and for 500 $5 \mathrm{mbar}(\mathrm{g}=1 \mathrm{~mm})$ at $100 \mathrm{~W}$. One can see that the pressure immo- 501 bilizes the values of $V_{\text {MAX }}$ and $V_{\text {bias }}$ independent of the gap value. 502 This indicates that the coaxial structure plays a major role in the 503 establishment of the plasma properties, much more than the 504 grounded external plate placed behind the slits. This information is 505 very important, since the discharge can be (almost) independently 506 adjusted with respect to the surface to treat.

To study the effect of pressure and gap theoretically, we 508 numerically simulated the plasma discharge corresponding to these 509 three cases, further presented and discussed in Sec. IV.

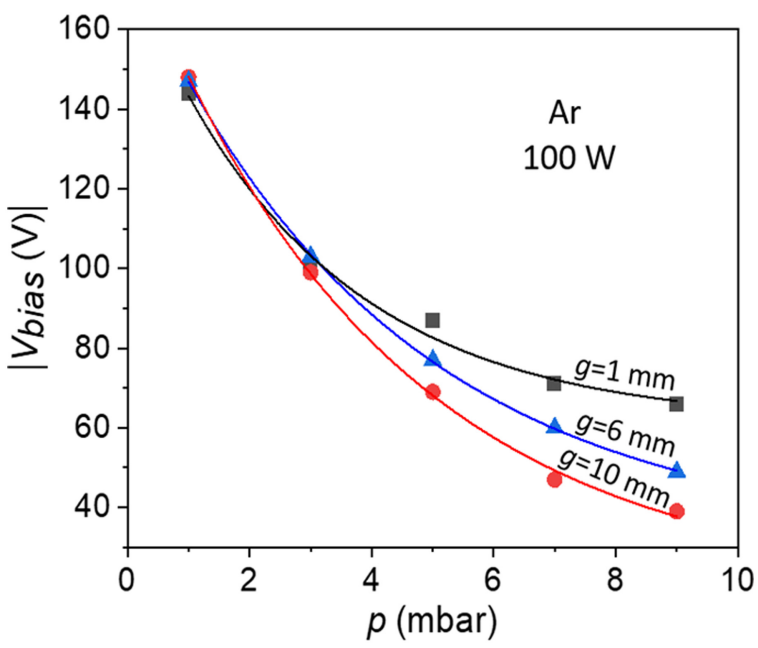

FIG. 9. Self-bias variation (absolute value) with gas pressure, for Ar plasma at $100 \mathrm{~W}(\mathrm{~g}=1,6$, and $10 \mathrm{~mm})$. 

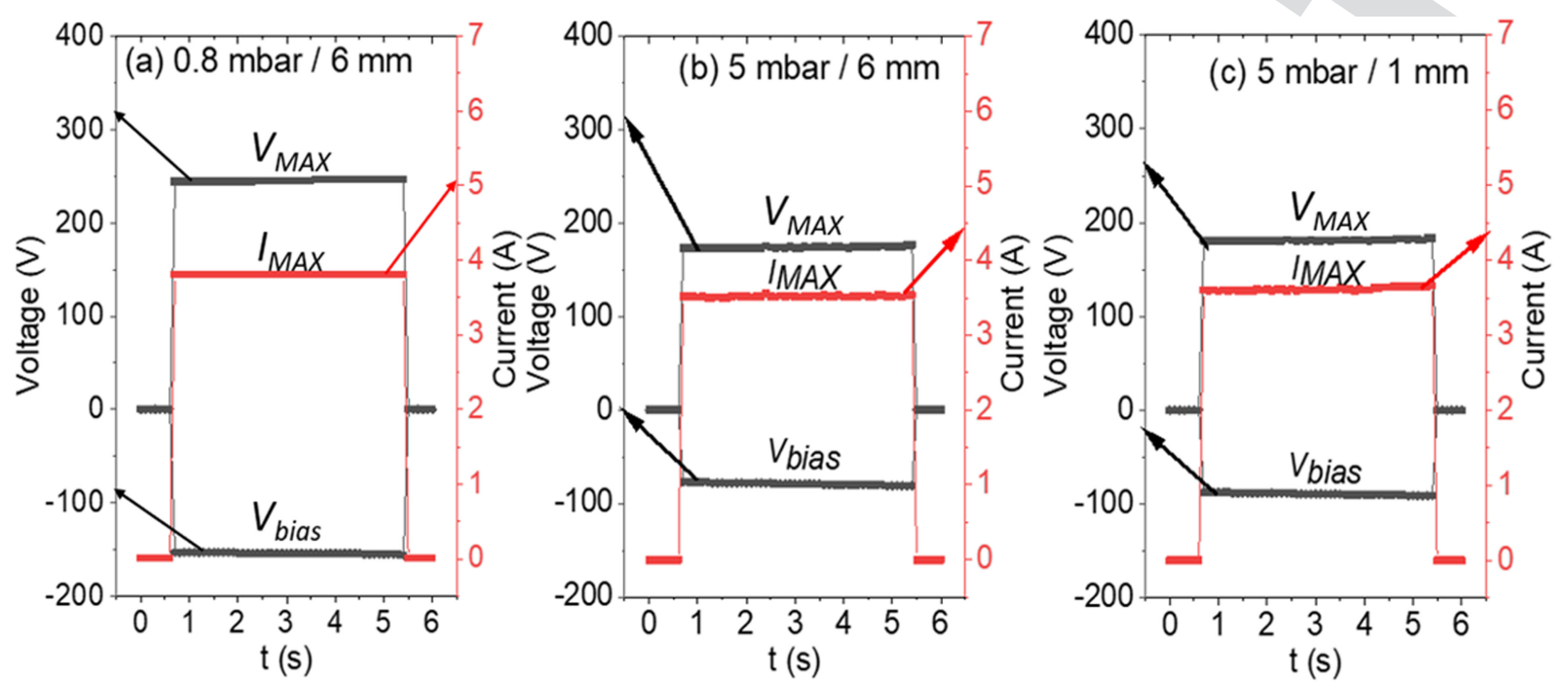

FIG. 10. Time stability of the discharge throughout the maxima of voltage, current $\left(V_{M A X}, I_{M A X}\right)$ and self-bias voltage $\left(V_{\text {bias }}\right)$ measured for (a) $0.8 \mathrm{mbar} / \mathrm{g}=6 \mathrm{~mm},(b)$ $5 \mathrm{mbar} / \mathrm{g}=6 \mathrm{~mm}$, and (c) $5 \mathrm{mbar} / \mathrm{g}=1 \mathrm{~mm}$ (reactor B: Ar plasma, $P_{\mathrm{RF}}=100 \mathrm{~W}$ ).

\section{IV. MODELING RESULTS AND DISCUSSION}

Numerical simulations were performed with the Plasimo software package, introduced in Sec. II B. The aim is to study this asymmetric CCCD, especially to evaluate the density and the flux of charged particles, the average electric potential, and the power dissipated by impinging ions striking the external plate, but also to get a deeper knowledge about the electron energy and other discharge parameters in order to optimize the system. Hence, the previous experimental results become easy to explain. This model has been run with several input parameters such as gas pressure, RF voltage, and geometrical parameters taken from the experiment. It should be noted here that for the Plasimo package, there is no difference between the geometry of the two reactors $\mathbf{A}$ and $\mathbf{B}$ because its geometry is reduced to the $2 \mathrm{D}$ cross section in the plane $(y, z)$ perpendicular to the cylinders axis $(x)$. The main numerical results are presented and discussed below. It should be noted that simulations of plasma self-organization in the axial direction are highly desirable to understand the nature of cluster formation, but this issue is out of the scope of the present paper.

\section{A. Effect of the gas pressure}

To study the effect of the gas pressure on discharge parameters, two simulations were performed for 0.8 and 5 mbar, using the experimental parameters shown in Fig. 10.

The input parameters were

(1) $V_{\text {MAX }}=245 \mathrm{~V}, V_{\text {bias }}=-155 \mathrm{~V}, p=0.8 \mathrm{mbar}$, and $g=6 \mathrm{~mm}$ [experimental parameters Fig. 10(a)],

(2) $V_{\text {MAX }}=176.5 \mathrm{~V}, \quad V_{\text {bias }}=-77 \mathrm{~V}, p=5 \mathrm{mbar}$, and $g=6 \mathrm{~mm}$ [experimental parameters Fig. 10(b)].

Figure 11 shows the $2 \mathrm{D}$ spatial distribution of the density of ions for the two pressures, namely 0.8 and 5 mbar. One can see that for both cases, a low density of ions is present between the tubes, on 541 each side of the center of the slits, but away from the slits, the density 542 of the plasma vanishes between the two cylinders (average density of 543 ions and electrons being close to zero). As we have already explained, 544 the two cylinders are very close to each other, which limits the forma- 545 tion of a dense plasma in the space separating them $(\delta=1.6 \mathrm{~mm}) .546$ The outer cylinder operates as a guard ring and should force the 547 plasma to form essentially inside the cavity created by the inner elec- 548 trode and outside the slits toward the external plate. So, between the 549 two tubes, there is a negligible loss in terms of power balance, which 550 was the initial goal when this geometry has been designed. 551

To analyze the ion effect on the plate, 1D curves of different 552 plasma parameters are plotted vs $y$ axis for the experimental 553

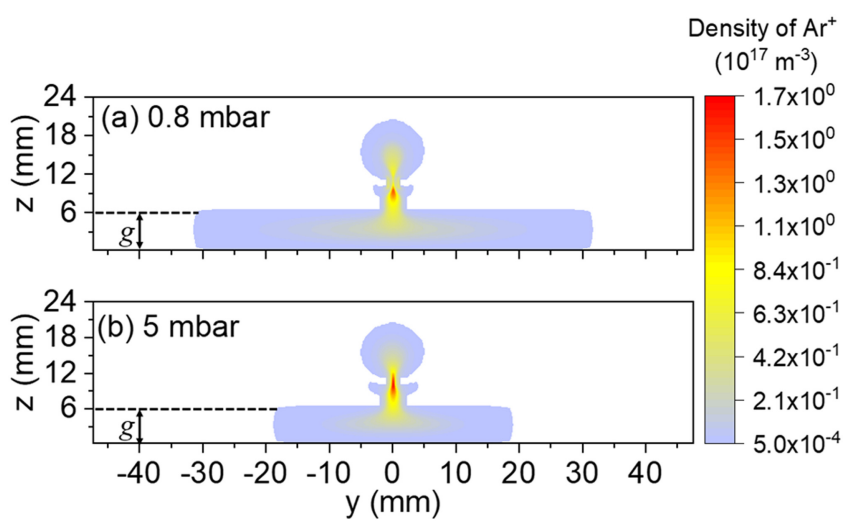

FIG. 11. 2D density of $\mathrm{Ar}^{+}$maps in the plane $(y, z)$ normal to the cylinders axis for (a) 0.8 and (b) $5 \mathrm{mbar}$, calculated by Plasimo $(\mathrm{g}=6 \mathrm{~mm})$. 

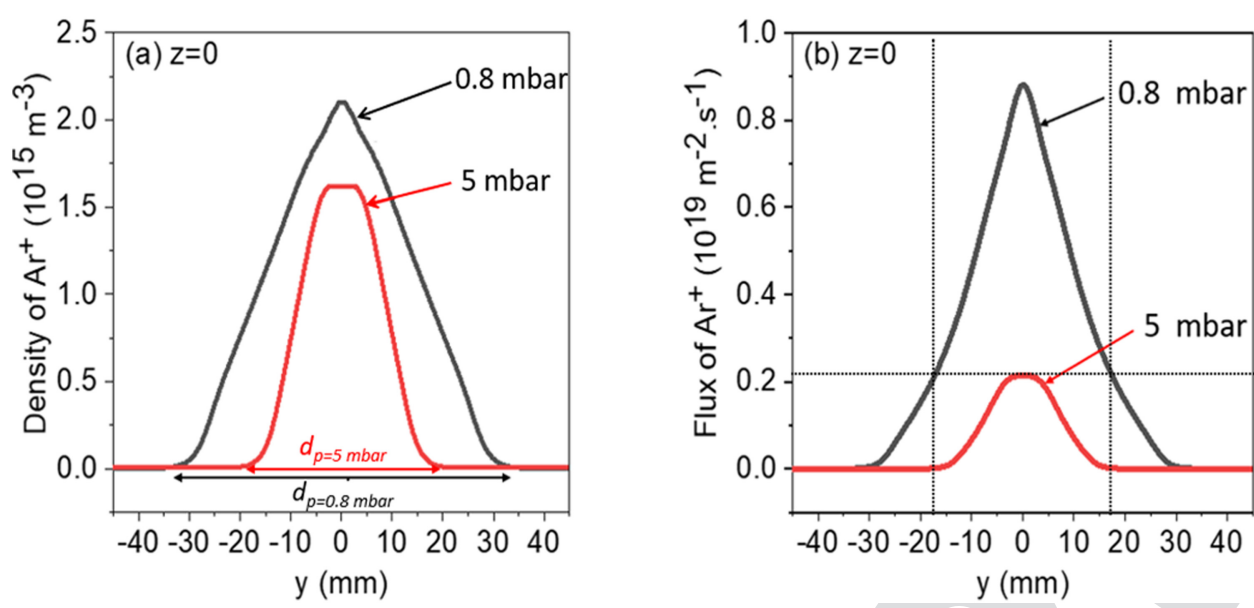

FIG. 12. Spatial distribution of (a) the density and (b) the flux of $\mathrm{Ar}^{+}$at the plate for 0.8 and $5 \mathrm{mbar}$, calculated by Plasimo. Same conditions as in Fig. 11.
554 position of the plate $(z=0$, on each side of the center of the slits,

Figures 12(a) and 12(b) show the spatial distribution of the density and the flux of $\mathrm{Ar}^{+}$, respectively, for the two pressures on the plate, just below the slit. In Fig. 12(a), the width of the plasma spreading onto the plate $d$ is defined for each pressure. The density and the ion flux are both higher for the lower pressure, here 0.8 mbar. Notice also that the ion flux is sharper at low pressure [Fig. 12(b)], its maximum being four times higher at $0.8 \mathrm{mbar}$ compared to 5 mbar.

To better understand the pressure effect on the discharge parameters close to the plate, Figs. 13(a) and 13(b) show the spatial distribution of the dissipated power by ions on the plate and their local velocity each side of the slit, for both pressures. It should be noted here that the power dissipated by ions is defined by the scalar product of the ion current density and the local electric field for different positions $(y, z=0)$, and the velocity of ions at the plate is calculated as the ratio of ion flux by the ion density at the plate.
As shown in Fig. 13, the power dissipated by ions and the 572 velocity of ions at the plate are both higher for 0.8 mbar. The dissi- 573 pated power density is very similar to the ion flux, being sharper at 574 $0.8 \mathrm{mbar}$ and having a maximum almost four times higher com- 575 pared to $5 \mathrm{mbar}$. This can be explained by the increased collision 576 frequency for higher pressures, so that an important fraction of the 577 power goes to the neutral gas and consequently the ion flux reach- 578 ing the plate is reduced. The ion mean velocity [Fig. 13(b)] clearly 579 indicates a collisional regime at 5 mbar with a maximum velocity at 580 the center of the slits and a ratio between the maxima of about 3, 581 and not 4 as found for the fluxes [Fig. 12(b)] and power density 582 [Fig. 13(a)]. That means the plasma density slightly increases with 583 the pressure, but the heating efficiency is definitely higher at low 584 pressure and it is important if the sheath is collisionless, as dis- 585 cussed in Sec. IV B.

Let us discuss the sheath formation on both sides, the inner 587 electrode and the plate (grounded). As said, for our configuration, 588 the powered electrode is the inner tube; its area $\left(A_{p}\right)$ is proportional 589
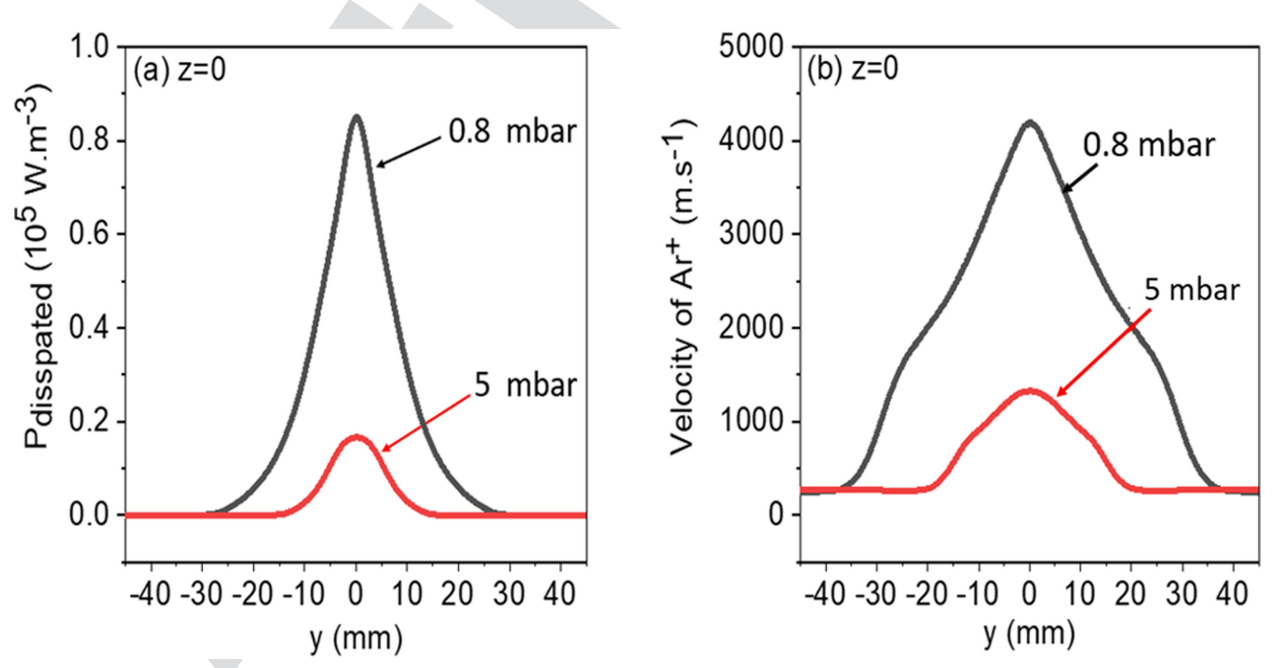

FIG. 13. Spatial distribution on the plate of (a) the power dissipated by $\mathrm{Ar}^{+}$ and (b) the mean velocity of $\mathrm{Ar}^{+}$for 0.8 and 5 mbar, calculated by Plasimo. Same conditions as in Fig. 11. 
590 to the internal radius and the length of the slit of the inner tube: $591 A_{p}=L_{i n} \times 2 \times \pi \times{ }_{i n}$.

The effective grounded electrode is the planar surface of the plate seen by the discharge, in the absence of any plasma formed in the space between the electrodes. This area $\left(A_{g}\right)$ is proportional to the distance $(d)$, with $d$ being the width of the plasma spreading onto the plate [see $d_{p=5 \mathrm{mbar}}$ and $d_{p=0.8 \mathrm{mbar}}$ in Fig. 12(a)]. $A_{g}$ is also proportional to the length of the slit of outer cylinder $\left(L_{\text {out }}\right)$ : $A_{g}=L_{\text {out }} \times d$.

As shown in Fig. 12(a), for $0.8 \mathrm{mbar}, d_{p=0.8 \mathrm{mbar}} \approx 62 \mathrm{~mm}$, but for $5 \mathrm{mbar}, d$ reduces to about $d_{p=5 \mathrm{mbar}} \approx 40 \mathrm{~mm}$, for the same other conditions. The increase of the distance $d$ is then related to the lateral expansion of the plasma out of the slit, which is facilitated by the decreasing the gas pressure.

For reactor $\mathbf{B}$ :

$$
A_{p}=L_{\text {in }} \times 2 \times p \times r_{\text {in }}=145 \times 2 \times p \times 5=4553 \mathrm{~mm}^{2},
$$

605

$$
A_{g}=L_{\text {out }} \times d=150 \times d .
$$

On the other hand, Fig. 14 shows the spatial distribution of the average electric potential $(\bar{V})$, along the vertical axis, starting from the plate $(z=0)$ up to the internal wall of the outer tube (grounded, $z=24 \mathrm{~mm}$ ), for the two pressures. The sheath thickness can be clearly evaluated from the potential drop in front of each electrode. Hence, the sheath thicknesses at the powered electrode $(\mathrm{z} \sim 20 \mathrm{~mm})$ and the grounded plate $(\mathrm{z} \sim 0 \mathrm{~mm})$ are indicated in Fig. 14 denoted by $s$ with a double index, the first one being related to the power " $p$ " or ground " $g$," and the second one to the pressure " 0.8 " and " 5 " being separated by a comma. Decreasing the pressure, the sheath width slightly increases at the powered electrode, but it decreases at the grounded one:

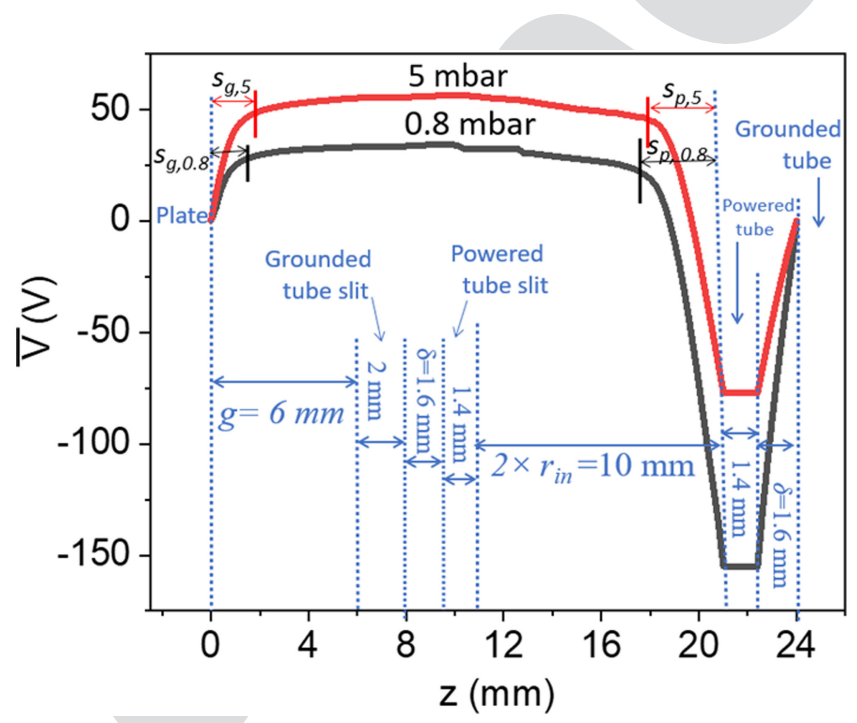

FIG. 14. Spatial distribution of the average electric potential, for Ar plasma for 0.8 and 5 mbar, for $y=0$, calculated by Plasimo. Same conditions as Fig. 11.

$p=0.8$ mbar: $s_{p}=3.5 \mathrm{~mm}, s_{g}=1.4 \mathrm{~mm}, d=62 \mathrm{~mm}$. By applying 619

Eq. (1): $V_{\text {bias }}=-164.7 \mathrm{~V}, \quad 620$ $p=5 \mathrm{mbar}: s_{p}=3.2 \mathrm{~mm}, s_{g}=1.8 \mathrm{~mm}, d=40 \mathrm{~mm}$. By applying 621 Eq. (1): $V_{\text {bias }}=-70.8 \mathrm{~V}$.

The decreasing of the pressure causes the expansion of the 623 plasma volume toward the grounded area, which is experimentally 624 verified for capacitive coupled discharge. ${ }^{10,19}$

According to the self-bias voltage formula [Eq. (1)], we found 626 values very close to those measured experimentally ( $\pm 6 \%$ to $8 \%$ ). 627 Therefore, this legitimates the good choice of the grounded elec- 628 trode surface and sheath thicknesses values estimated from the sim- 629 ulations, for each case. This is taken as a first rough validation of 630 the model. In other words, the guard ring (outer cylinder) plays a 631 minor role in the discharge energy balance, but it keeps the 632 maximum ionization (plasma density) in the slit's region. Indeed, 633 from Fig. 14, one can undoubtedly see that the outer electrode 634 plays well the role of guard ring since the powered sheath thickness 635 is larger than the distance between the cylinders $\left(s_{p}>\delta\right)$; That 636 means it is not possible to form a plasma in the space between the 637 two cylinders, consistent with our initial design. 638

In addition, Fig. 15 shows the temporal evolution of the 639 average electron energy during some RF periods. The average elec- 640 tron energy for $0.8 \mathrm{mbar}$ is $\epsilon_{\mathrm{e}} \sim 10.2 \pm 3.5 \mathrm{eV}$, which is higher than 641 that for 5 mbar, where it is only $\varepsilon_{e} \sim 4.3 \pm 0.5 \mathrm{eV}$.

From these two well-chosen simulations, for two pressures 643 (0.8 and 5 mbar) corresponding to experimental conditions, the 644 values of the surface seen by the discharge on the grounded elec- 645 trode (external plate) and the sheaths' thicknesses are estimated 646 through the spatial distribution of the average density of ions and 647 electrons; we verified the expansion of the plasma toward the 648 grounded electrode with the decrease of the pressure, which is in 649

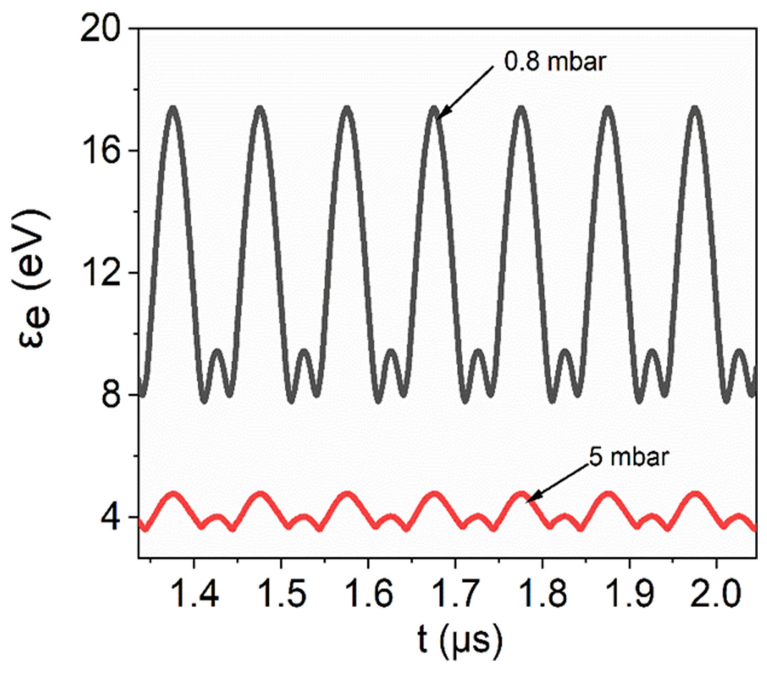

FIG. 15. Variation of the average electron energy for Ar plasma, for 0.8 and 5 mbar during some RF periods, calculated by Plasimo. Same conditions as in Fig. 11. 
Density of $\mathrm{Ar}^{+}$ $\left(10^{17} \mathrm{~m}^{-3}\right)$

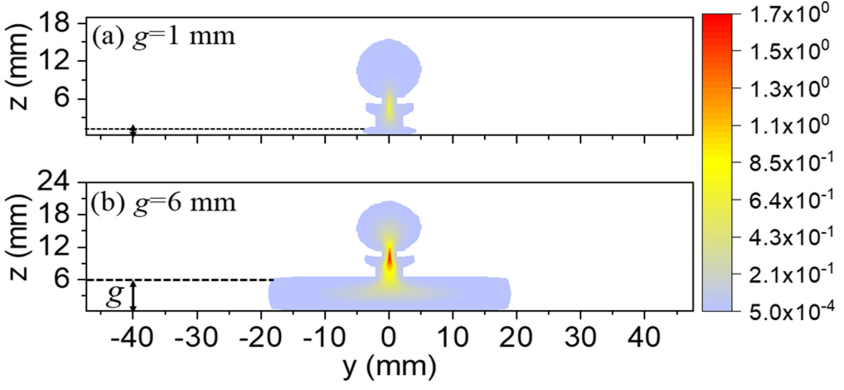

FIG. 16. $2 \mathrm{D}$ density of $\mathrm{Ar}^{+}$maps in the plane $(y z)$ normal to the cylinders axis, for (a) $g=1$ and (b) $g=6 \mathrm{~mm}$, calculated by Plasimo. ( $p=5 \mathrm{mbar}$ ).

650 line with other experimental observations for a capacitive coupled 651 discharge. $^{10,19}$

652 At this stage, it clearly appears the system is more efficient for 653 the surface treatment if the working pressure is the lowest possible. 654 In our case, reducing the pressure from 5 to 0.8 mbar enhances the 655 plasma effect onto the plate in terms of density, flux, ion mean 656 velocity, and power density dissipation at the surface, by at least a 657 factor of 3, at the same RF power.

\section{B. Effect of the reactor/plate gap distance}

To study the effect of the gap distance between the reactor and the external plate, simulations were performed for a fixed pressure $p=5 \mathrm{mbar}$, but for two well-chosen gap values $g=1$ and $6 \mathrm{~mm}$. The input parameters were

(1) $V_{\text {MAX }}=176.5 \mathrm{~V}, \quad V_{\text {bias }}=-77 \mathrm{~V}, p=5 \mathrm{mbar}$, and $g=6 \mathrm{~mm}$ [experimental parameters Fig. 10(b)],

(2) $V_{M A X}=180 \mathrm{~V}, V_{\text {bias }}=-87 \mathrm{~V}, p=5 \mathrm{mbar}$, and $g=1 \mathrm{~mm}$ [experimental parameters Fig. $10(\mathrm{c})]$.
Figure 16 shows the 2D spatial distribution of the density of 667 ions for $g=1$ and $6 \mathrm{~mm}$. Similarly, away from the slits, the plasma 668 does not form between the tubes. One can see that the increase of 669 the gap from 1 to $6 \mathrm{~mm}$ decreases the width of the plasma spread- 670 ing onto the plate: the increase of the distance $d$ is then closely 671 related to the lateral expansion of the plasma, which is facilitated 672 by the available drift space when increasing the gap.

673

As shown in Fig. 16, for the region of plasma bulk (between 674 the two slits), the density of ions is higher for the gap of $6 \mathrm{~mm} 675$ than that for the short gap of $1 \mathrm{~mm}$. The increase of plasma 676 density with increasing of the gap was verified for capacitive 677 coupled plasma: ${ }^{33}$ the increase of the gap from 1 to $4 \mathrm{~cm}$ markedly 678 increases the density of electrons, corresponding to an obstructed 679 discharge. ${ }^{41}$ The discharge glows easier with larger gaps.

680

As previously, to compare the effect on the plate, the curves 681 are plotted vs one coordinate perpendicular to the slits, $y$ (at $z=0$ ). 682 Figures 17 (a) and 17 (b) show the spatial distribution on the plate 683 of the density and the flux of $\mathrm{Ar}^{+}$for each considered gap. The 684 density and the flux are much higher (about three times) for the 685 lower gap, $g=1 \mathrm{~mm}$, but the treated area is much narrower, in the 686 ratio of the distance $d$ seen by the plasma, 30/7.2 = 4 times larger in 687 terms of ion flux.

In addition, Fig. 18 shows the spatial distribution of the power 689 density dissipated by ions and their mean velocity at the plate. One 690 can see that both plasma parameters are higher and sharper for the 691 shortest gap.

By calculations, the integral of each curves of Fig. 18(a) 693 divided by the width at half maximum (which represents the width 694 over which the energy is deposited), we notice a ratio of four times 695 greater for the smallest gap $(1 \mathrm{~mm})$; the calculations are presented 696 on the figure for each case. There is a huge interest to reduce the 697 gap which act as reducing the width of the plasma slab and made 698 the process as a linear plasma curtain lying on a surface.

699

To evaluate the model capabilities, an estimation of the 700 sheaths is made similar to the one performed in Sec. IV A. 701 Figure 19 shows the average potential along the vertical axis from 702 the plate $(z=0)$ to the inner (upper side) of the RF electrode (to 703 $z=16 \mathrm{~mm}$ for $g=1 \mathrm{~mm}$ and to $z=21 \mathrm{~mm}$ for $g=6 \mathrm{~mm}$ ), for both 704
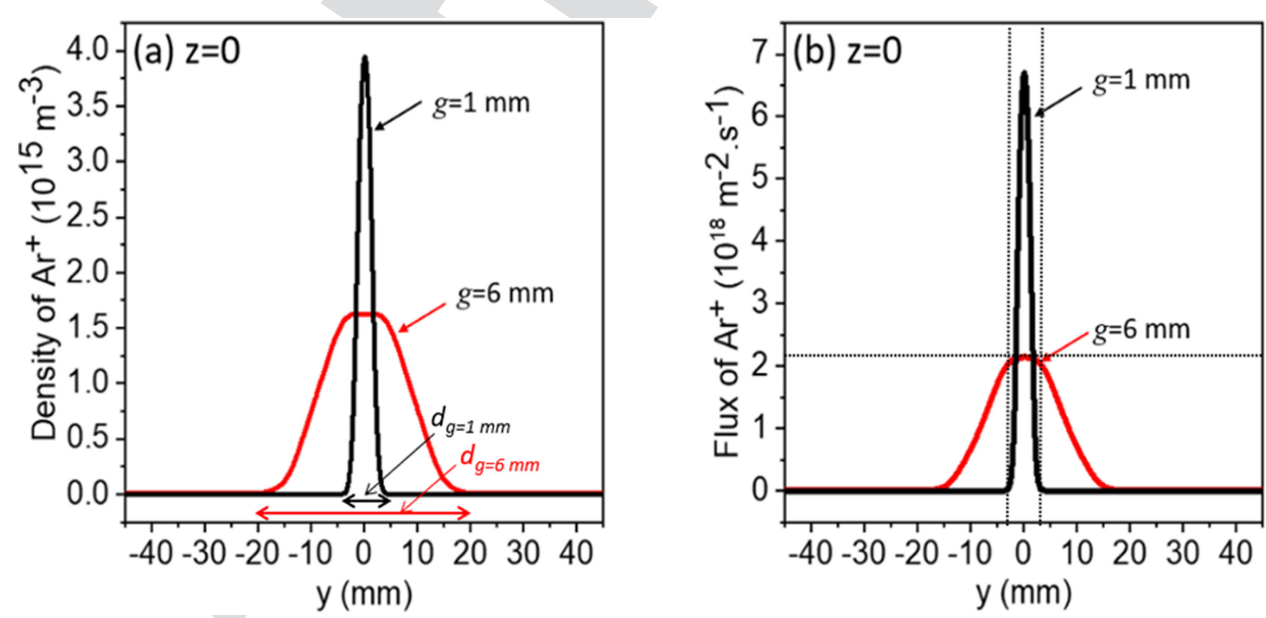

FIG. 17. Spatial distribution of (a) the density and (b): the flux of $\mathrm{Ar}^{+}$at the plate for $\mathrm{g}=1$ and $6 \mathrm{~mm}$, calculated by Plasimo. Same conditions as in Fig. 16. 


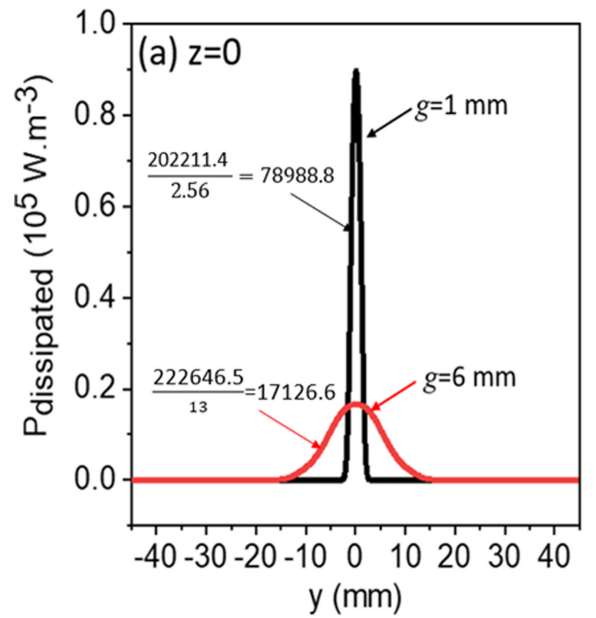

705 sin

714 for each gap value,

$715 g=1 \mathrm{~mm}: d=10 \mathrm{~mm}, s_{p}=6.4 \mathrm{~mm}, s_{g}=0.9 \mathrm{~mm}$. By applying Eq. 716 (1): $V_{\text {bias }}=-72.3 \mathrm{~V}$. To be compared to measured $-87 \mathrm{~V}$,

$717 \mathrm{~g}=6 \mathrm{~mm}: d=40 \mathrm{~mm}, s_{p}=3.2 \mathrm{~mm}, s_{g}=1.8 \mathrm{~mm}$. By applying

718 Eq. (1): $V_{\text {bias }}=-70.8$ V. To be compared to measured $-77 \mathrm{~V}$.

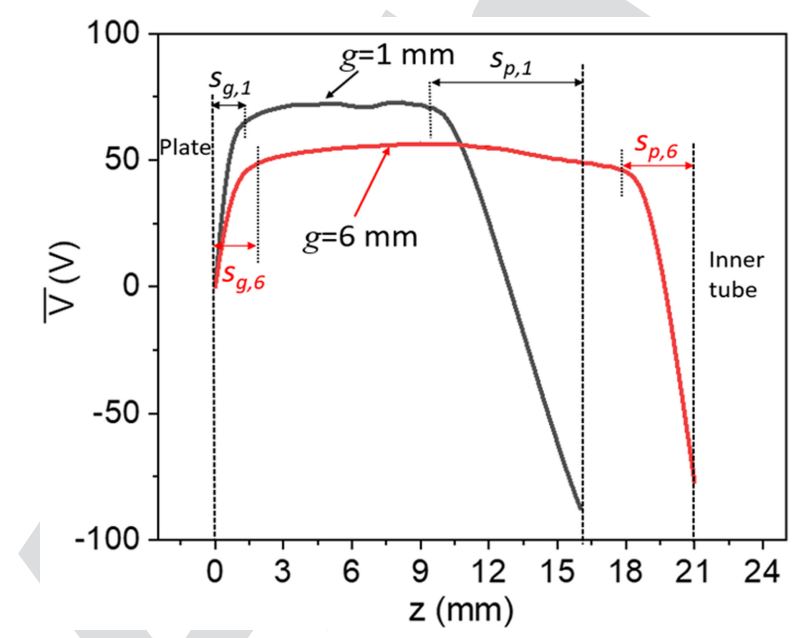

FIG. 19. Spatial distribution of the average electric potential along the normal at
the plate, for $y=0$, for $g=1$ and $6 \mathrm{~mm}$, calculated by Plasimo. Same conditions

FIG. 19. Spatial distribution of the average electric potential along the normal at
the plate, for $y=0$, for $g=1$ and $6 \mathrm{~mm}$, calculated by Plasimo. Same conditions as in Fig. 16.

simulated situations ( $g=1$ and $6 \mathrm{~mm}$ ). One can see that the poten. hicknesses $\left(s_{p}\right.$ and $\left.s_{g}\right)$ for each case. The second index of $s$ in g. 19 corresponds in this time to the value of the gap.

As shown in Sec. III B (Fig. 9), the self-bias voltage is slightly

From the spatial distribution of the ion density and the

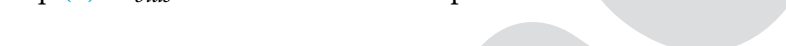
(a)

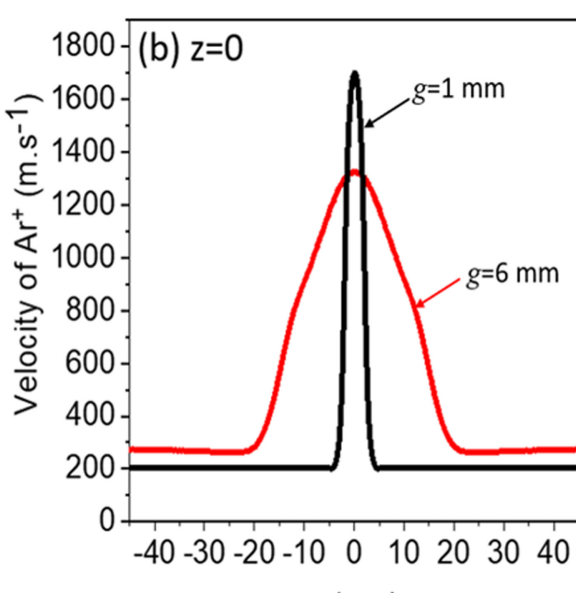

$\mathrm{y}(\mathrm{mm})$

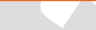

According to the self-bias voltage formula, we found values in 719 the same order of magnitude to those measured experimentally, 720 but the error exceeds $17 \%$ for the shortest gap $(g=1 \mathrm{~mm})$. This is 721 an indication that approaching the discharge closer to the plate 722 (ground), the outer slit (grounded as well) starts to play in the RF 723 circuit so its role as electrode cannot be neglected anymore. 724 However, within the error margin, the coarse result legitimates the 725 estimation of the active electrode surfaces and the sheath thick- 726 nesses provided by the numerical simulations. We take this as a 727 second rough validation of the model.

To finalize this discussion, Fig. 20 shows the temporal evolu- 729 tion of the average electron energy for these last two cases. This 730 energy is, in average, $\epsilon_{\mathrm{e}} \sim 6.8 \pm 1.1 \mathrm{eV}$ and $\epsilon_{\mathrm{e}} \sim 4.3 \pm 0.5 \mathrm{eV}$ for 731 $g=1$ and $6 \mathrm{~mm}$, respectively. A similar result has been already 732 reported, showing that the increase of the gap distance from 1 to 733 $4 \mathrm{~cm}$ leads to a decrease of the electron temperature in the 734 plasma.

From these two last simulations ( $g=1$ and $6 \mathrm{~mm}$, Fig. 20), the 736 values of the surface of the plate playing the role of grounded elec- 737 trode and the sheath thicknesses have been estimated through the 738 spatial distribution of the ion density and the average plasma 739 potential. In addition, for a short gap of $1 \mathrm{~mm}$, the density, the 740 flux, the velocity of ions, and the energy they dissipate at the plate 741 are all higher than those of the case of $6 \mathrm{~mm}$. Moreover, the plasma 742 slab becomes much narrower, very suitable for localized surface 743 treatment. Therefore, it is better to operate this discharge very close 744 to the surface to treat (external plate, here $1 \mathrm{~mm}$ ).

At this stage, it is also interesting to validate the effect of gas 746 obtained in Sec. IV A but this time for the shortest gap (1 mm). 747 Hence, the two panels (a) and (b) of the Fig. 21 show, respectively, 748 the spatial distribution of the flux and the power density dissipated 749 by ions, for two pressures 5 and $1.4 \mathrm{mbar}$, and a fixed short gap 750 $g=1 \mathrm{~mm}$. One can see that both plasma parameters are higher for 751 the lowest pressure (1.4 mbar). So again, the system is more effi- 752 cient for the surface treatment if the working pressure is kept as 753 low as possible. The lower limit is due to the ability to glow the dis- 754 charge, and it depends, as experimentally found, on the type of gas 755 used much more than the size of the reactor. 


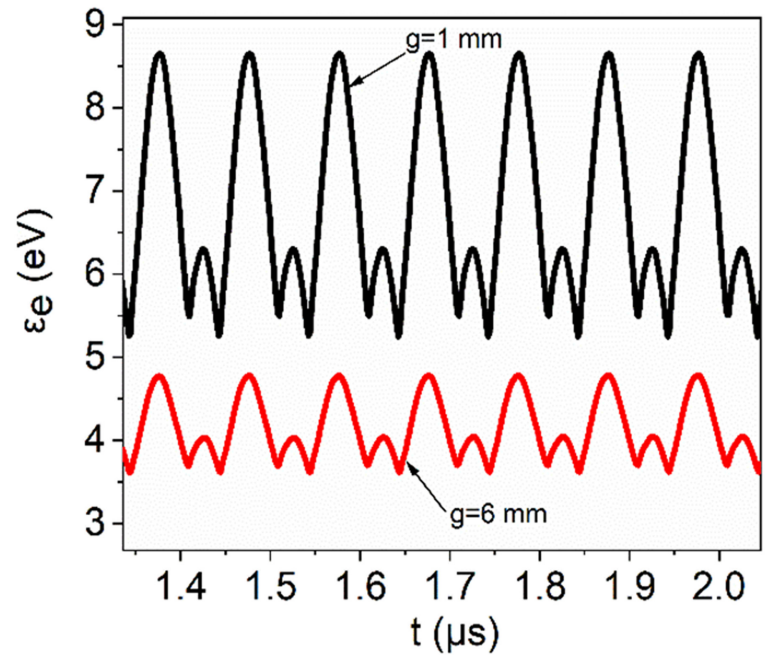

FIG. 20. Variation of the average electron energy for Ar plasma for some RF periods, for $\mathrm{g}=1$ and $6 \mathrm{~mm}$, calculated by Plasimo. Same conditions as in Fig. 16.

It should be noted here also that for the shortest gap $(1 \mathrm{~mm})$, even numerically it is difficult to develop a plasma. Plasimo modeling package failed (could not converge) with RF power of $100 \mathrm{~W}$ (same RF power for other simulated cases, Fig. 10) and for the pressures below 1 mbar. To overcome this limitation, we have simulated the case of $1.4 \mathrm{mbar}$ (Fig. 21) and not the exact value of pressure used in the experiment.

As expected by its design, this cylindrical capacitive coupled discharge design produces two important ion sheaths. The one for the powered hallow electrode is usual, but the second one formed in front of the grounded plate appears to be the key for surface treatment. It is governed by the large electron losses imposed to the system by construction. The formation of an important sheath in front of the plate was the initial goal, leading to an efficient acceleration of the ions toward the plate surface and so doing effective ion assistance. Notice that this CCCD operates with only one RF power 772 supply. Moreover, the ion flux to the plate can be tuned by chang- 773 ing the pressure, or simply the distance between the electrodes and 774 the plate.

Even if the present experimental study has been performed 776 with a metal plate, the discharge is certainly effective on the treat- 777 ment of dielectric materials, since the RF sheaths drain an equiva- 778 lent amount of charged particles on each electrode, overall each 779 period. Moreover, this plasma system can be very effective for large 780 area surface treatment when moving one (i.e., either the discharge 781 or the plate) relative to the other in a direction perpendicular to 782 the slits. These characteristics of the proposed CCCD give it a high 783 versatility.

\section{CONCLUSION}

In this work, a novel design for capacitive coupled argon 786 plasma is presented, formed essentially by two cylindrical coaxial 787 electrodes and called cylindrical capacitive coupled discharge 788 (CCCD). If both cylinders are equipped with a slit, and these slits 789 are aligned, then it is possible to use this device for surface treat- 790 ment of a flat object or plate placed just under the slits. Two 791 reactor configurations have been designed and characterized, with 792 different lengths (the second configuration was twice longer than 793 the first one), and they operate properly. This can be taken as a 794 first proof of the possible scaling-up of this system.

A linear RF plasma is formed inside the cavity of the inner 796 cylinder, and it spreads out through the slits, forming a plasma slab 797 between the reactor and the plate. It has a stable operation over a 798 relatively wide range of pressures $(0.8-50 \mathrm{mbar}$ ) or RF power (typi- 799 cally $<1.4 \mathrm{~kW})$. Experimentally, the self-bias voltage measured was 800 negative, as expected. Obviously, the self-bias voltage increases, in 801 absolute value, when increasing the RF injected power and decreas- 802 ing the gas pressure.

Using the fluid modeling (Plasimo package), the values of the 804 surface seen by the plasma on the grounded plate, acting as a 805 ground electrode, could be evaluated. From the model, the lateral 806 width under the slits receiving the charged particles and the sheath 807 thicknesses were estimated for all studied conditions. The optimized 808

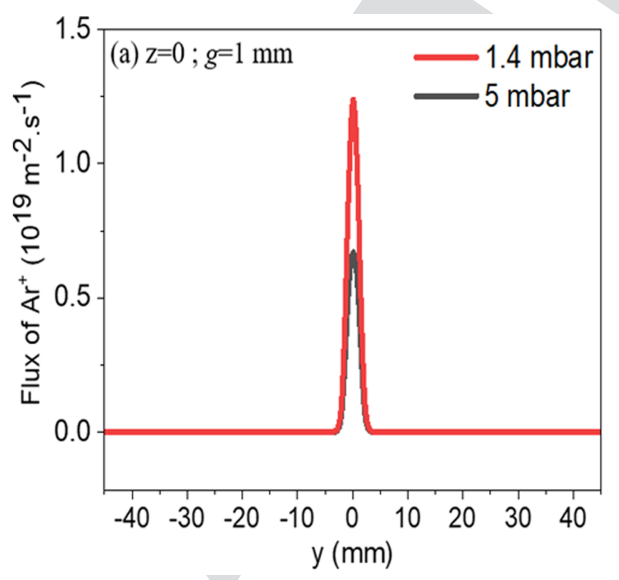

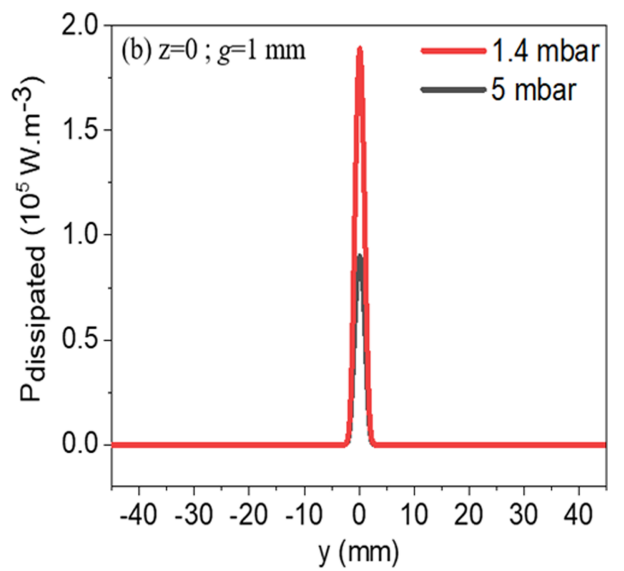

FIG. 21. Spatial distribution of (a) the flux of $\mathrm{Ar}^{+}$and (b) the power dissipated by $\mathrm{Ar}^{+}$at the plate for $\mathrm{g}=1$, and for 1.4; and $5 \mathrm{mbar}$, calculated by Plasimo, with $\mathrm{V}_{\text {MAX }} N_{\text {bias }}$ are $245 \mathrm{~V} /-135 \mathrm{~V}$ for $1.4 \mathrm{mbar}$ and $180 \mathrm{~V} /-87 \mathrm{~V}$ for $5 \mathrm{mbar}$. 
809 operation conditions require low gas pressure and the plate very 810 close to the reactor slits.

The present configuration is very useful for the surface 812 heating applications and for specific conditions (non-stationary 813 cluster formation); it can be used for ion assistance on the surface 814 under the slits.

815 Moreover, in this work, the plate was grounded, but it is possi816 ble to bias it as desired, positively or negatively, depending on the 817 followed purpose. Also, it can develop a self-bias voltage if the plate 818 is made in an isolator material. As a perspective, the use of this 819 plasma could be extended following the power transferred from the 820 plasma to the plate, simply by tracking the increase of the plate 821 temperature. This would be an easy means to evaluate the efficiency 822 of this novel discharge configuration in terms of ion flux and 823 surface treatment.

\section{DATA AVAILABILITY}

825 826 from the corresponding author upon reasonable request.

\section{REFERENCES}

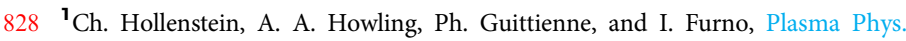
829 Control. Fusion 57, 014010 (2015).

$830{ }^{2}$ M. A. Lieberman and A. J. Lichtenberg, in Principles of Plasma Discharges and 831 Materials Processing, 2nd ed. (John Wiley \& Sons Inc., 2005), ISBN: 8329780471720010.

$833^{3}$ P. Chabert and N. Braithwaite, Physics of Radiofrequency Plasmas (Cambridge 834 University Press, Cambridge, 2011).

835 'R. W. Gould, Phys. Lett. 11, 236 (1964).

$836{ }^{\mathbf{5}}$ H. R. Koenig and L. I. Maissel, IBM J. Res. Dev. 14, 168 (1970).

$837{ }^{6}$ M. A. Lieberman and A. J. Lichtenberg, Principle of Plasma Discharges and 838 Materials Processing (Wiley, 1994).

Q5 839 7K. Köhler, J. W. Coburn, D. E. Horne, and E. Kay, J. Appl. Phys. 57, 59 (1985).

840 8. W. Coburn and E. Kay, J. Appl. Phys. 43, 4965 (1972).

$841{ }^{9}$ M. J. Cooke and J. Pelletier, Appl. Phys. Lett. 53, 19 (1988).

$842{ }^{10}$ R. Hytry and D. Boutard-Gabillet, Appl. Phys. Lett. 69, 752 (1996).

843 '1Y. Catherine and P. Couderc, Thin Solid Films 144, 265 (1986).

844 12 B. G. Heil, U. Czarnetzki, R. P. Brinkmann, and T. Mussenbrock, J. Phys. D: 845 Appl. Phys. 41, 165202 (2008).

$846{ }^{13}$ M. V. Alves, M. A. Lieberman, V. Vahedi, and C. K. Birdsall, J. Appl. Phys. 69, 8473823 (1991).
${ }^{14}$ C. M. Horwitz, J. Vac. Sci. Technol. A 1, 60 (1983).

${ }^{15}$ E. Kawamura, M. A. Lieberman, A. J. Lichtenberg, and E. A. Hudson, J. Vac. 849 Sci. Technol. A 25, 1456 (2007).

${ }^{16}$ A. F. Alexandrov, A. Y. El Sammoni, V. A. Godiak, and A. A. Kuzovnikov, in 851 Proceedings of the VIII ICPIG, Vienna, Austria $(\mathbf{\square}, 1967)$, p. 165.

${ }^{17}$ H. Baránková, L. Bardos, and K. Silins, ECS J. Solid State Sci. Technol. 5(9), 853 N57-N60 (2016).

${ }^{18}$ M. A. Lieberman and S. E. Savas, J. Vac. Sci. Technol. A 8, 1632 (1990). 855

${ }^{19}$ J. Upadhyay, J. Peshl, S. Popovic, A.-M. Valente-Feliciano, and L. Vuskovic, 856 AIP Adv. 8, 085008 (2018). 857

${ }^{20}$ G. J. M. Hagelaar, "Modeling of microdischarges for display technology," 858 Ph.D. thesis (Eindhoven University of Technology, 2000). 859

${ }^{21}$ G. J. M. Hagelaar, Brief Documentation of BOLSIG+ Version 03/2016 860 (Laboratoire Plasma et Conversion dOEnergie (LAPLACE) (Universit Paul 861 Sabatier, 2016), Vol. 118.

${ }^{22}$ See www.lxcat.net for the Viehland database. 863

${ }^{23}$ Handbook of Physical Quantities, edited by I. S. Grigoriev and E. Z. Meilikhov 864 (CRC Press, Boca Raton, FL, 1997). 865

${ }^{24}$ J. T. Gudmundsson and A. Hecimovic, Plasma Sources Sci. Technol. 26, 866 123001 (2017).

${ }^{25}$ Y.-X. Liu et al., Phys. Plasmas 24, 073512 (2017). ${ }^{\mathbf{2 6}}$ V. I. Kolobov, Glow Discharges: Stratification Encyclopedia of Plasma 869 Technology (Taylor and Francis, London, 2017).

${ }^{27}$ V. I. Kolobov, J. Phys. D: Appl. Phys. 39, R487 (2006).

${ }^{28}$ Y. B. Golubovskii et al., Tech. Phys. 59, 1787 (2014).

${ }^{29}$ A. S. Penfold and J. A. Thornton, Czech J. Phys. 23, 431435 (1973).

${ }^{30} \mathrm{H}$. C. J. Mulders et al., IEEE Trans. Plasma Sci. 36, 1380 (2008).

${ }^{31}$ Y. P. Raizer, Gas Discharge Physics (Springer, Berlin, 1991).

${ }^{32}$ See https://plasimo.phys.tue.nl/ for

${ }^{33}$ Y. Ohtsu and H. Fujita, Jpn. J. Appl. Phys. 43, 795799 (2004).

${ }^{34}$ V. I. Kolobov et al., J. Phys. D: Appl. Phys. 53, 25LT01 (2020). ${ }^{35}$ D. I. N. E. Sébastien, Effet de la Fréquence Dans les Décharges VHF sur les 879 Caractéristiques des Plasmas Utilisés Pour le Dépôt de Silicium Microcristallin 880 (Université Paris Sud, Paris, 2006).

${ }^{36} \mathrm{M}$. Tanișli, N. Șahin, and S. Demir, Pramana 89(3), 36 (2017).

${ }^{37}$ M. Tanışli, N. Şahin, S. Demir, and S. Mertadam, Plasma Phys. Rep. 45(4), 883 376-386 (2019).

${ }^{38}$ A. M. Ahadi, T. Trottenberg, S. Rehders, T. Strunskus, H. Kersten, and 885 F. Faupel, Phys. Plasmas 22(8), 083513 (2015).

${ }^{39}$ R. W. Boswell and A. Bouchoule, Plasma Chem. Plasma Process. 8(1), 53887 (1988).

${ }^{40}$ V. A. Godyak, R. B. Piejak, and B. M. Alexandrovich, IEEE Trans. Plasma Sci. 889 19, 660 (1991).

${ }^{41}$ J. T. Gudmundsson, Plasma Sources Sci. Technol. 29, 113001 (2020). $\quad 891$

${ }^{42}$ K. Bera et al., J. Appl. Phys. 129, 053304 (2021). \\ Q6}

(1)

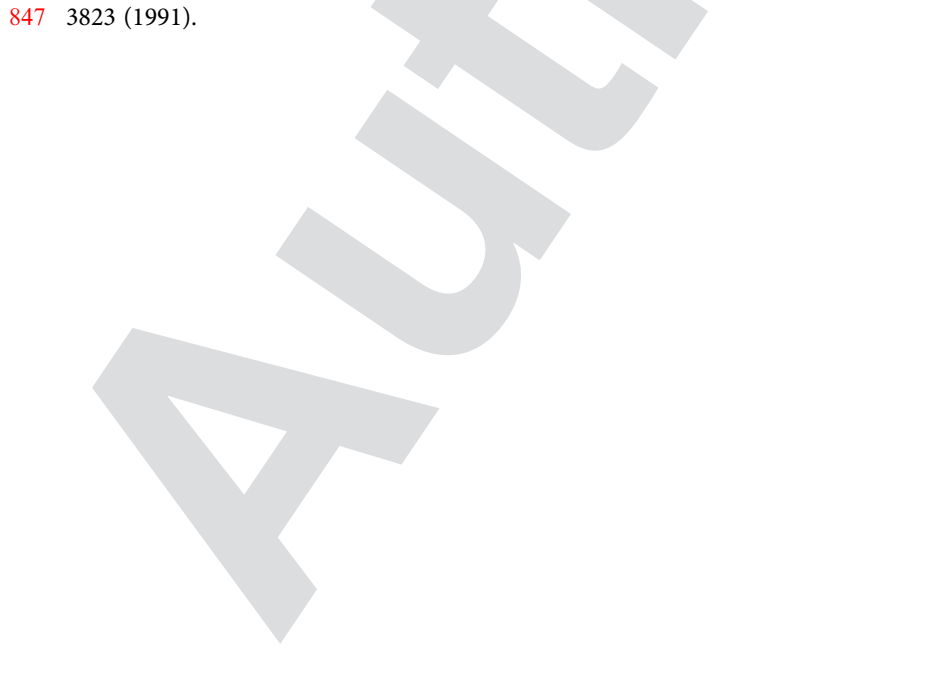

